\title{
Experimental and Numerical Investigation of Tire Tread Wear on Block Level
}

\author{
Felix Hartung ${ }^{1}$, Mario Alejandro Garcia ${ }^{1}$, Thomas Berger ${ }^{1}$, Michael Hindemith ${ }^{2}$, Matthias Wangenheim ${ }^{2} \mathbb{D}$ \\ and Michael Kaliske ${ }^{1, *}$ \\ 1 Institute for Structural Analysis, Technische Universität Dresden, 01062 Dresden, Germany; \\ felix.hartung@tu-dresden.de (F.H.); mario.garcia@tu-dresden.de (M.A.G.); \\ thomas.berger@tu-dresden.de (T.B.) \\ 2 Institute of Dynamics and Vibration Research, Leibniz Universität Hannover, 30167 Hannover, Germany; \\ hindemith@ids.uni-hannover.de (M.H.); wangenheim@ids.uni-hannover.de (M.W.) \\ * Correspondence: michael.kaliske@tu-dresden.de
}

Citation: Hartung, F.; Garcia, M.A.; Berger, T.; Hindemith, M.;

Wangenheim, M.; Kaliske, M.

Experimental and Numerical Investigation of Tire Tread Wear on Block Level. Lubricants 2021, 9, 113. https://doi.org/10.3390/

lubricants 9120113

Academic Editor: Maria Dolores Bermudez

Received: 6 October 2021

Accepted: 16 November 2021

Published: 23 November 2021

Publisher's Note: MDPI stays neutral with regard to jurisdictional claims in published maps and institutional affiliations.

Copyright: (C) 2021 by the authors. Licensee MDPI, Basel, Switzerland. This article is an open access article distributed under the terms and conditions of the Creative Commons Attribution (CC BY) license (https:// creativecommons.org/licenses/by/ $4.0 /)$.

\begin{abstract}
Tread wear appears as a consequence of friction, which mainly depends on surface characteristics, contact pressure, slip velocity, temperature and dissipative material properties of the tread material itself. The subsequent description introduces a wear model as a function of the frictional energy rate. A post-processing as well as an adaptive re-meshing algorithm are implemented into a finite element code in order to predict wear loss in terms of mass. The geometry of block models is generated by image processing tools using photographs of the rubber samples in the laboratory. In addition, the worn block shape after the wear test is compared to simulation results.
\end{abstract}

Keywords: wear; friction; finite element method; adaptive meshing; linear friction test; image processing

\section{Introduction}

The tire is the only contact between vehicles and road surfaces and, thus, it has an important function: it must transmit all forces and moments in all spatial directions as reliably as possible. It is known that in tire design, compromises must be found between functional goals, in particular (wet) braking, mileage, rolling resistance, and noise emission [1]. In addition, wear particle emission comes more and more into focus of legislative institutions [2]. Looking closer into the construction of tires with its many rubber layers, textile plies, and steel belts, the one that is in contact with the road is the tire tread, which is usually geometrically subdivided into blocks of different size or circumferential ribs. Hence, all contact forces and moments are transmitted via the tire tread and it is the tread suffering wear and emitting wear particles. In literature, the wear rate of rubber compounds is often described by a function proportional to the frictional power dissipated during contact, while the frictional power is directly dependent on the local sliding speed, the local contact pressure, and the coefficient of dynamic friction (compare e.g., [3-5]). The force that can be transmitted between tire and road is determined by the local friction characteristics which is composed of static friction in the sticking zone of the contact patch and dynamic friction in the sliding zone [6]. Besides the road surface quality and the rubber material formulation, it is known that the dynamic coefficient of friction between rubber and a rough surface depends on a number of parameters, in particular local contact pressure, sliding velocity, and the contact temperature are of great importance. These parameters are a direct consequence of the tire construction and the maneuvers in which the tire undergoes.

Linear friction testers (in this case HiLiTe [7] was used) are a common methodology to assess friction between tire tread blocks and (road) surface sections [8]. These devices are used to quasi-steadily investigate characteristics of the coefficient of sliding friction in a wide parameter range, i.e., contact pressure, sliding speed, and in some cases also ambient temperature are varied but held constant during a single test run. The range of 
parameters is defined to cover all relevant situations (e.g., ABS braking or cornering) the test is intended to represent.

In this paper, it is described for the first time how a linear friction tester is used to quasi-steadily characterize not only tread block friction but also tire tread wear, feeding Finite Element (FE) based tread block friction, and wear simulations. The tread block model demands for a number of parameter sets that are direct outcomes of these experiments, in particular the friction characteristics in terms of local contact pressure and sliding speed and the wear characteristics in terms of contact pressure, sliding speed, and local coefficient of friction. Furthermore, the tread block model consists of a material description covering all important effects, in this case a viscoelastic rubber material model, whose parameters are assessed by uni-, biaxial tension, and pure shear tests as well as the Dynamic Mechanical Analysis (DMA). A self-evident but important constraint is that friction and wear experiments are performed in a parameter range covering the full parameter space of the simulations. Experimentally, quasi-steadiness is achieved by conditioning of the block, i.e., the block slides a number of runs before measurement starts, to gain an initial wear shape as well as attaining a steady-state temperature [9]. In FE simulations, wear results in loss of material, even if parameters and simulations are performed quasi-steadily. One method to take volume loss into account is recurring re-meshing of the block geometry (see, e.g., $[3,4,10,11])$. In this paper, a novel method is presented to perform mesh generation and re-meshing based on automatic image processing of the tread block shape during linear friction tester experiments. Hence, a good agreement between simulations and friction and wear tests can be achieved.

\section{Materials and Methods}

\subsection{Experimental Setup}

For the experiments we made use of the High-speed Linear Tester (HiLiTe, see Figure 1) at the Institute of Dynamics and Vibration Research (IDS) at Leibniz Universität Hannover.

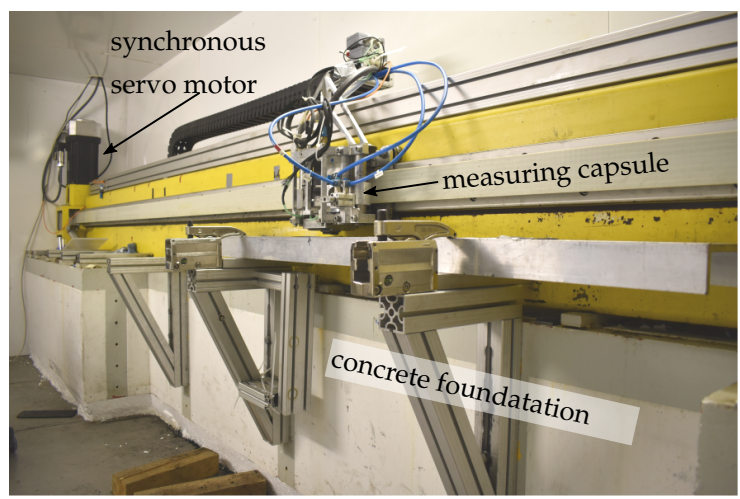

(a)

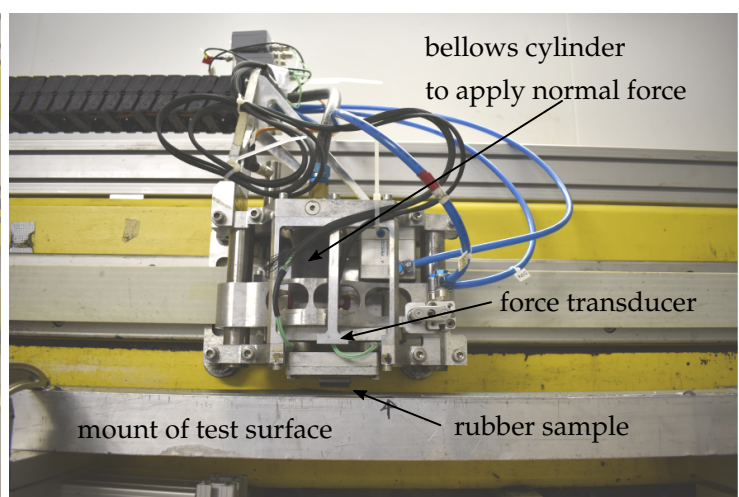

(b)

Figure 1. High-Speed Linear Tester (HiLiTe) for friction and wear measurements. (a) Overall view, (b) Detail lateral view.

A tread block sample is pressed onto a test track with a defined normal force and slides with a defined velocity profile relative to the track. With this type of motion, the sliding process between tire tread and road surface of a rolling tire under slip is replicated. Hence, HiLiTe's sliding velocity corresponds to the velocity difference between tire circumferential speed and vehicle speed, e.g., during acceleration or braking. The sliding speed range covered by HiLiTe in the current configuration is up to $10 \mathrm{~m} / \mathrm{s}$. The normal force between tire tread bock and counter surface can be selected up to $1000 \mathrm{~N}$. This enables testing passenger car tread blocks as well as truck or aircraft treads in dependence of the sample size and, in particular, its contact area. The normal force during a test is set in relation to the pneumatic tire pressure to be replicated multiplied with the nominal contact area of the tread block, which is typically at least $20 \times 20 \mathrm{~mm}^{2}$ at a maximum height of $10 \mathrm{~mm}$ to limit block distortion. HiLiTe is located in a climate chamber, where temperature and humidity 
can be controlled in order to be able to test in winter as well as in summer conditions. A vast variety of test tracks can be used, such as asphalt or concrete, both in wet and dry conditions, as well as snow and ice. In this test campaign, a sand paper grit of 60 is utilized to study the wear behavior of a tread block compound with the advantage of comparably high abrasiveness with corresponding short testing times. Another advantage of this type of surface is the homogeneity of the contact pressure distribution, as the roughness topography features are small in comparison to the tread block sample. Furthermore, the dominant wear mechanism on sand paper is abrasion, and other wear mechanisms like fatigue or thermal degradation play only a secondary role. After bringing the tread block into contact with the counter surface and building up the normal force (with a pistonless bellows cylinder), the sample accelerates on an aluminum sheet. Thereby, wear is avoided in the acceleration phase and it is possible to evaluate steady-state friction and wear as soon as the sample enters the grinding paper track with steady velocity. In this set-up, the abrasive track length is set to $200 \mathrm{~mm}$. Deceleration is performed in air after the sample has left the grinding paper track at its end.

Directly in the tread block sample holder, normal force and tangential friction force are measured, by which the coefficient of friction is calculated. Force sensors are arranged as dynamometer array to compensate for moments in the sensor set-up. The belt moving the sample holder carriage is driven by a servo motor, whose internal rotational sensors are utilized for obtaining tread block position and speed. In addition, the height of the sample can be measured inductively with the help of a Linear Variable Differential Transformer (LVDT) sensor. The acquisition rate of all measurement data is $10 \mathrm{kHz}$. In this study, sample wear is gravimetrically computed by measuring the sample mass before and after a test by a precision balance by a resolution of $0.1 \mathrm{mg}$. The result is wear rate (mass loss) per sliding distance.

\subsection{Test Procedure}

The main goal of the experiments performed in this study is the acquisition of quasisteady characteristics of friction and wear rate of a tire tread block in terms of sliding speed and mean contact pressure. In this context, quasi-steadiness refers to keeping sliding velocity, normal load, and environmental conditions including surface tidiness constant during one test run [12]. Furthermore, the need for a uniform contact pressure distribution under the tread block can be identified, because wear rate as well as coefficient of friction are strongly dependent on the (local) contact pressure. Hence, all blocks are conditioned before testing. The conditioning of the blocks is done by sliding $6 \times 1 \mathrm{~m}$ distance on the sand paper used in the tests. The speed is set to $1 \mathrm{~m} / \mathrm{s}$ and the contact pressure to $1.2 \mathrm{~N} / \mathrm{mm}^{2}$. The evolution of the tread block geometry can be seen in Figure 2 in the side view.

The bottom edge takes an "S-shaped" form [13], with the leading edge being worn stronger than the trailing edge. Under normal and tangential load, this wear shape finally results in a homogeneous contact pressure distribution. In consequence, both wear rate and coefficient of friction can be considered in steady state with a good repeatability and reproducibility, with a typical standard deviation of the measurement results of $9 \%$ (friction on asphalt tracks) and 12\% (wear rate on asphalt tracks). The testing maneuver is conducted as follows (see Figure 3):

A Sample is set down into contact with the aluminum sheet on track, with no relative motion until all vibrations are stopped. Normal force is applied via a pneumatic bellows cylinder. The resting time before start of motion is approximately $0.5 \mathrm{~s}$. Then, acceleration with $a=5 g=49.05 \mathrm{~m} / \mathrm{s}^{2}$ to the intended speed still on the aluminum track. The length of the aluminum track is large enough to ensure that the acceleration phase of the sample is finished before coming into contact with the abrasive test surface; 
B Leaving the aluminum sheet and coming into contact with the abrasive surface. Short phase of vertical dynamics because of the step down to the track with respective effects in signal of coefficient of friction;

C Overcoming the static friction of the sample on the abrasive counter surface. For the transfer of results to rolling friction, assessment of the static friction coefficient is essential due to the large contribution of the sticking zone to the tangential forces in the tire footprint. Transitioning from sticking to sliding requires some time, in particular steady-state temperatures and block deflection have to be established. In this transition period, the coefficient of friction decreases slightly. The transition time lag constants are specific for the respective frictional contact and affected by materials, surface topographies, and potential third media in contact. Subsequently, a quasi-steady period of sliding friction occurs with a constant level of coefficient of friction, which is evaluated as the steady-state coefficient of friction;

D At the end of the test track, the sample is decelerated and leaves the contact.

sliding direction

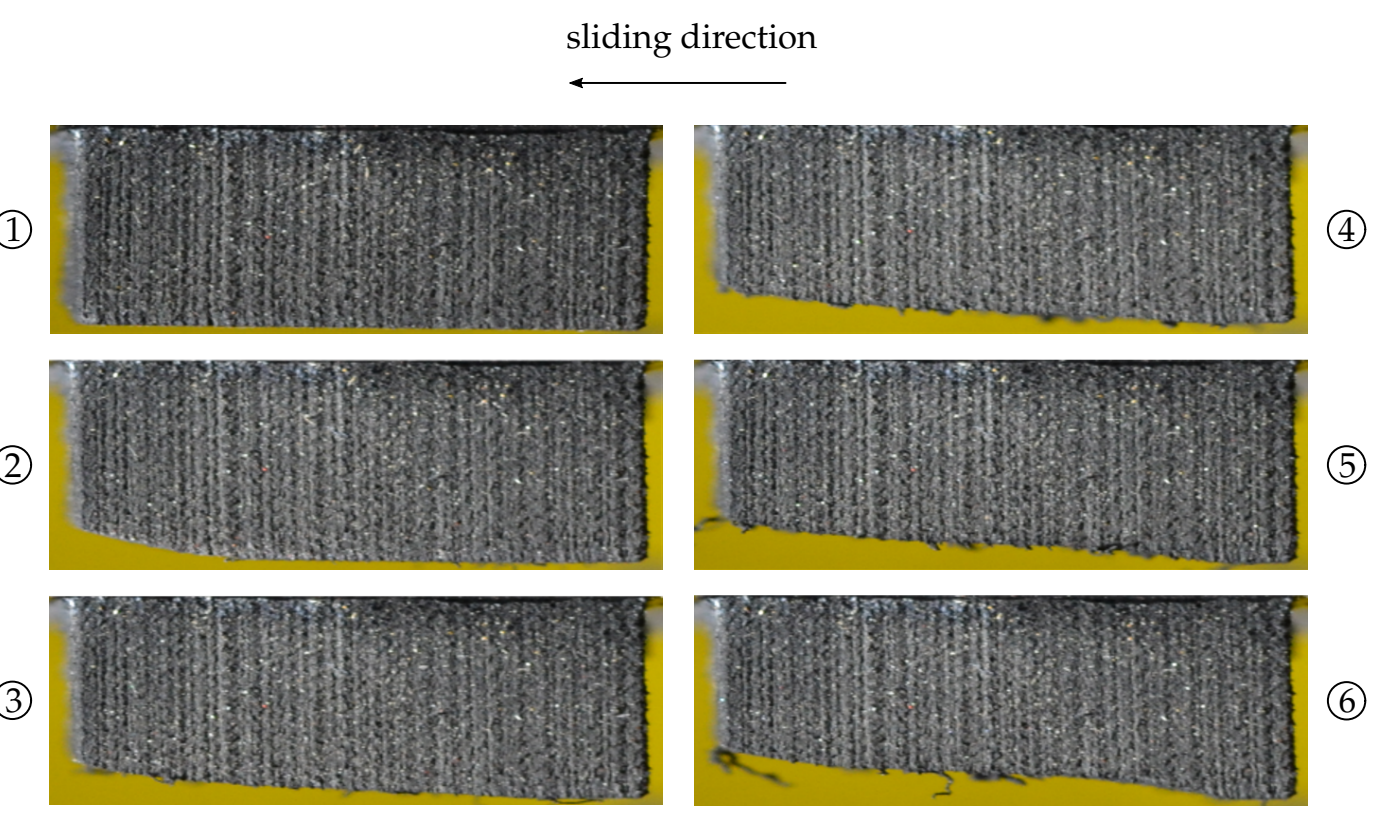

Figure 2. Pre-conditioning.

This approach enables us to define the sliding distance as exact as possible and ensures that abrasion occurs under quasi-steady conditions without interferences by tread block longitudinal dynamics. The friction and wear tests are repeated with five conditioned samples with very good repeatability as shown in Figure 3.

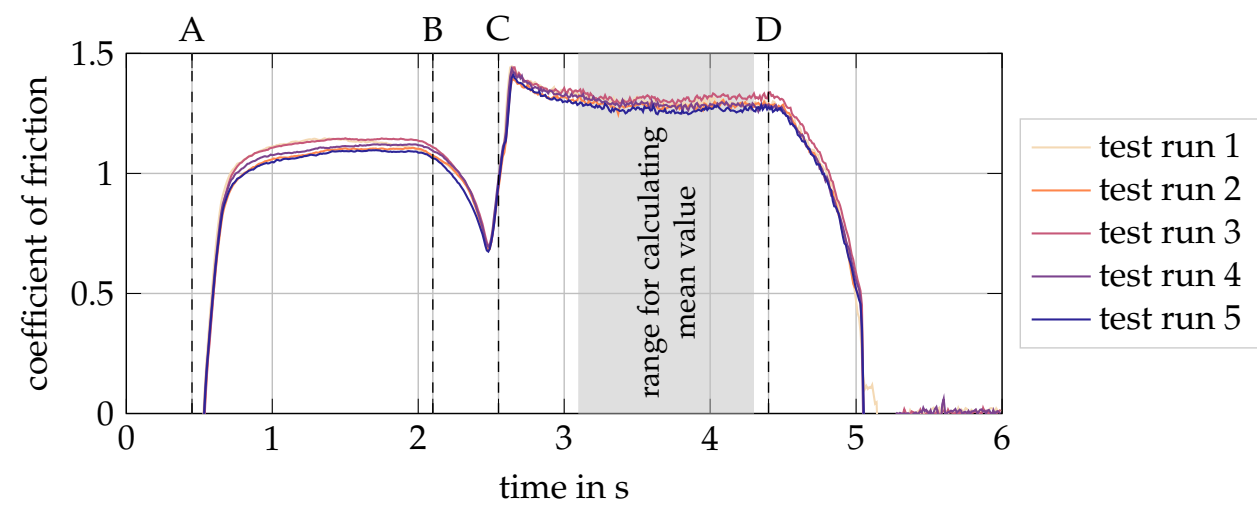

Figure 3. Test repetitions at $p=0.4 \mathrm{~N} / \mathrm{mm}^{2}$ and $v=50 \mathrm{~mm} / \mathrm{s}$. 
After each single test run, the track is cleaned from rubber debris with compressed air and a soft brush. In case of material transfer to the track, rubber layers can be cleaned by manual wiping with uncured natural rubber (NR). The samples are disassembled from the test rig and weighed every 1 to 20 cycles (depending on the abrasion rate) after blowing loose particles from the sample surface by compressed air. Since the coefficient of friction on the aluminum sheet adjusts with a time delay during the acceleration phase, the acceleration time $t=\frac{50 \mathrm{~mm} / \mathrm{s}}{49.05 \mathrm{~m} / \mathrm{s}^{2}} \approx 0.001 \mathrm{~s}$ does not coincide with the time until the friction coefficient is steady state between points A and B in Figure 3.

\subsection{Material Model for Rubber}

Rubber material is characterized by non-linear elasticity at large strains (hyperelasticity), time dependent effects due to viscoelasticity, and consists of an isochoric (volumepreserving) and a volume changing contribution. To capture a wide range of frequencies, the generalized MAXWELL model, which is shown in Figure 4, is chosen for the isochoric part.

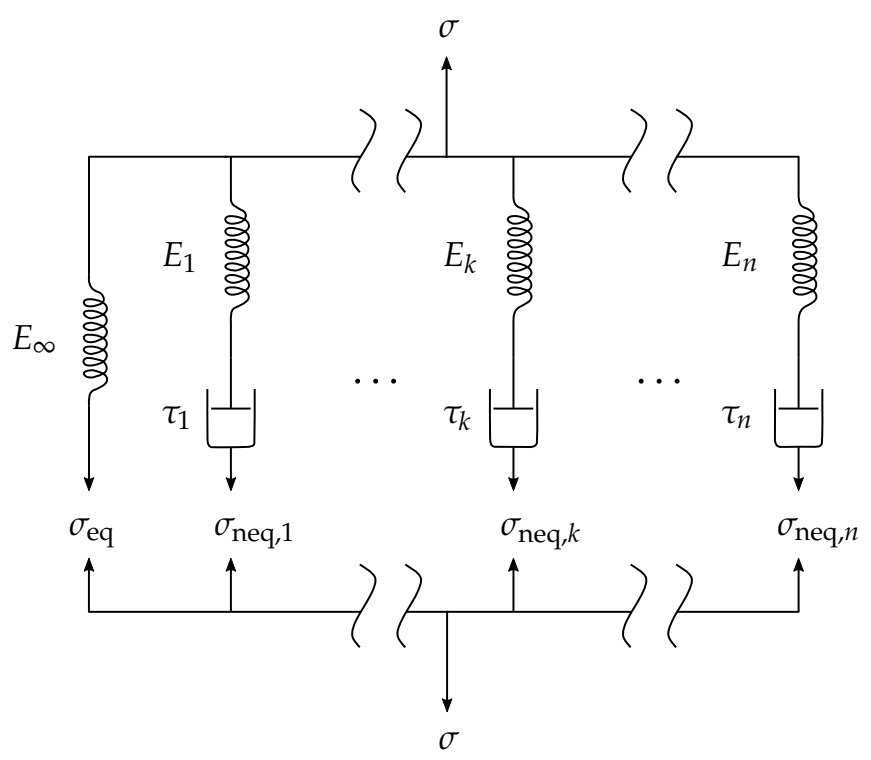

Figure 4. Material rheology for isochoric part (generalized MAXWELL model).

The first branch of the generalized MAXWELL model (equilibrium branch) consists of a single spring with the so-called long-term stiffness $E_{\infty}=\gamma_{\infty} E_{0}$ (stiffness at quasistatic loads), where $E_{0}$ represents the instantaneous stiffness of the entire rheological model (stiffness at very high dynamic loads). The remaining branches are MAXWELL elements (non-equilibrium branches) in parallel. Each MAXWELL element is composed of a spring with the spring stiffness $E_{k}(k=1, \ldots, n)$ and a dashpot with the dynamic viscosity $\eta_{k}=E_{k} \tau_{k}$, where $\tau_{k}$ is the relaxation time of dashpot $k$. In small strain theory, the one-dimensional strain of a MAXWELL element

$$
\varepsilon=\varepsilon_{e}+\varepsilon_{v}
$$

is the sum of the elastic and the viscous strain, whereas the stress in both, spring and dashpot, are identical. By inserting $\varepsilon_{e}=\sigma_{\text {neq }, k} / E_{k}$ and $\dot{\varepsilon}_{v}=\sigma_{\text {neq, }, k} / E_{k} \tau_{k}$ in the first time derivative of Equation (1), the differential equation

$$
E_{k} \dot{\varepsilon}=\dot{\sigma}_{\text {neq, } k}+\frac{\sigma_{\text {neq }, k}}{\tau_{k}}
$$


of MAXWELL element $k$ is derived. Equation (2) is a linear rate equation. By inserting the EULERian representation regarding stresses

$$
\sigma_{k}=\hat{\sigma}_{k} \exp (i \omega t)
$$

and strains

$$
\varepsilon=\hat{\varepsilon} \exp [i(\omega t-\delta)],
$$

with $i:=\sqrt{-1}$, stress amplitude $\hat{\sigma}_{k}$, strain amplitude $\hat{\varepsilon}$ and $\delta$ as phase shift between stress and strain response in Equation (2), the differential equation

$$
i \omega \tau_{k} E_{k} \hat{\varepsilon}=\hat{\sigma}_{k} \exp (i \delta)\left(1+i \omega \tau_{k}\right)
$$

is solved for an oscillation in the complex plane. This formulation defines the complex modulus

$$
E_{k}^{*}=\frac{\hat{\sigma}_{k}}{\hat{\varepsilon}} \exp (i \delta)=E_{k} \frac{i \omega \tau_{k}}{1+i \omega \tau_{k}}
$$

of MAXWELL element $k$ within the generalized MAXWELL model. The complex modulus $E_{k}^{*}=E_{k}^{\prime}+i E_{k}^{\prime \prime}$ can be decomposed into a real contribution (storage modulus) and an imaginary part (loss modulus). By use of the normalized stiffness factors $\gamma_{k}=E_{k} / E_{0}$ with $\gamma_{\infty}+\sum_{k=1}^{n} \gamma_{k}=1$, the storage modulus

$$
E^{\prime}=\gamma_{\infty} E_{0}+\sum_{k=1}^{n} \frac{\left(\omega \tau_{k}\right)^{2}}{1+\left(\omega \tau_{k}\right)^{2}} \gamma_{k} E_{0}
$$

and the loss modulus

$$
E^{\prime \prime}=\sum_{k=1}^{n} \frac{\omega \tau_{k}}{1+\left(\omega \tau_{k}\right)^{2}} \gamma_{k} E_{0}
$$

of the entire generalized MAXWELL model are determined as function of frequency $f=2 \pi / \omega$.

For the three-dimensional constitutive law, a continuum model based on large strain theory is applied. The small strain formulation is expanded by introducing the deformation gradient $\underline{\boldsymbol{F}}=\partial x / \partial x$ that maps point coordinates of the continuum from reference $X$ to current configuration $x$. The deformation gradient of one MAXWELL element at a material point

$$
\underline{\boldsymbol{F}}=\underline{\boldsymbol{F}}_{\mathrm{vol}} \underline{\boldsymbol{F}}_{\mathrm{iso}}=\left(J^{-1 / 3} \underline{\mathbf{1}}\right)\left(\underline{\boldsymbol{F}}_{e} \underline{\boldsymbol{F}}_{v}\right)
$$

can be split multiplicatively into a volumetric and an isochoric part, whereby the latter is composed of an elastic and a viscoelastic contribution. In the considered case, $J=\operatorname{det} \underline{\boldsymbol{F}}>0$ stands for the ratio of volume change, which is nearly one for rubber-like materials. The right CAUCHY-GREEN tensor $\underline{\boldsymbol{C}}=\underline{\boldsymbol{F}}^{\mathrm{T}} \underline{\boldsymbol{F}}$ and left CAUCHY-GREEN tensor $\underline{\boldsymbol{b}}=\underline{\boldsymbol{F}} \underline{\boldsymbol{F}}^{\mathrm{T}}$ describe the strain in the reference and the current configuration, respectively. In contrast to the multiplicative split of $\underline{\boldsymbol{F}}$, the stress state of the generalized MAXWELL model in the reference configuration is described by the second PIOLA-KIRCHHOFF stress tensor

$$
\underline{S}=\underline{S}_{\mathrm{vol}}+\underline{S}_{\mathrm{iso}}=\underline{S}_{\mathrm{vol}}+\underline{S}_{\mathrm{eq}}+\sum_{k=1}^{n} \underline{S}_{\mathrm{neq}, k}
$$

additively. In analogy to the stress tensor, the tangent modulus

$$
\underline{\mathbb{C}}=\underline{\mathbb{C}}_{\mathrm{vol}}+\underline{\mathbb{C}}_{\mathrm{iso}}=\underline{\mathbb{C}}_{\mathrm{vol}}+\underline{\mathbb{C}}_{\mathrm{eq}}+\sum_{k=1}^{n} \underline{\mathbb{C}}_{\mathrm{neq}, k}
$$


in the reference configuration is obtained. Based on linear viscoelasticity, the differential equation for MAXWELL element $k$

$$
\gamma_{k} \underline{\dot{S}}_{\mathrm{eq}}=\underline{\dot{S}}_{\mathrm{neq}, k}+\frac{1}{\tau_{k}} \underline{\boldsymbol{S}}_{\text {neq, } k}
$$

is derived from Equation (2). Equation (12) is described in the reference configuration to preserve objectivity. The non-equilibrium stress of MAXWELL element $k$

$$
\begin{aligned}
\underline{S}_{\text {neq }, k}\left(t_{j+1}\right) & =\gamma_{k} \int_{t=0}^{t_{j+1}} \exp \left(-\frac{t_{j+1}-t}{\tau_{k}}\right) \frac{\mathrm{d} \underline{S}_{\mathrm{eq}}(t)}{\mathrm{d} t} \mathrm{~d} t \\
& =\exp \left(-\frac{\Delta t}{\tau_{k}}\right) \underline{\boldsymbol{S}}_{\text {neq, } k}\left(t_{j}\right)+\gamma_{k} \frac{1-\exp \left(-\Delta t / \tau_{k}\right)}{\Delta t / \tau_{k}}\left[\underline{S}_{\mathrm{eq}}\left(t_{j+1}\right)-\underline{S}_{\mathrm{eq}}\left(t_{j}\right)\right],
\end{aligned}
$$

with the time increment $\Delta t=t_{j+1}-t_{j}$, is computed by a recurrence relation solving the convolution integral (see [14]). Note that the application of Equation (13) reduces the computational costs significantly, because the current stress state is computed from the stresses at the previous time step $t_{j}$. The derivative of the isochoric part of the second PIOLAKIRCHHOFF stress tensor with respect to the corresponding right CAUCHY-GREEN tensor

$$
\underline{\mathbb{C}}_{\text {iso }}\left(t_{j+1}\right)=2 \frac{\partial \underline{S}_{\text {iso }}\left(t_{j+1}\right)}{\partial \underline{\boldsymbol{C}}_{\text {iso }}\left(t_{j+1}\right)}=\left[1+\sum_{k=1}^{n} \frac{1-\exp \left(-\Delta t / \tau_{k}\right)}{\Delta t / \tau_{k}}\right] \underline{\mathbb{C}}_{\text {eq }}\left(t_{j+1}\right)
$$

yields the three-dimensional formulation of the tangent modulus of the rheology model in Figure 4 for large strains in the reference configuration. Thus, the isochoric material tangent results from the current isochoric material tangent of the equilibrium branch at $t_{j+1}$ due to the recursive formulation of Equation (13). In order to obtain the material tensor

$$
\underline{\mathbb{C}}_{\mathrm{eq}}=4 \frac{\partial^{2} \Psi_{\mathrm{eq}}}{\partial{\underline{C_{\mathrm{iso}}}}^{2}},
$$

the second order derivative of the strain energy density function of the equilibrium part with respect to the strain tensor $\underline{C}_{\mathrm{iso}}$ is required [15]. The YEOH model

$$
\Psi_{\mathrm{YEOH}}=C_{10}(I-3)+C_{20}(I-3)^{2}+C_{30}(I-3)^{3}
$$

with its stiffness parameters $C_{10}, C_{20}$ and $C_{30}$ is applied for the equilibrium branch as well as for the elastic part of each MAXWELL element [16]. For the equilibrium branch, the first invariant

$$
I=\operatorname{tr} \underline{C}_{\text {iso }}=\operatorname{tr} \underline{b}_{\mathrm{iso}}=\lambda_{\mathrm{iso}, 1}^{2}+\lambda_{\mathrm{iso}, 2}^{2}+\lambda_{\mathrm{iso}, 3}^{2}
$$

is equal to the trace of the isochoric left or right CAUCHY-GREEN strain tensor of the entire generalized MAXWELL model. In Equation (17), $\lambda_{\text {iso, } i}$ with $i=1,2,3$ represent the isochoric principle strains. The strain energy density function of the volumetric contribution

$$
\Psi_{\mathrm{vol}}=\frac{\kappa_{0}}{2}(J-1)^{2}
$$

depends on the bulk modulus $\kappa_{0}$ and the ratio of volume change $J$ [15]. The corresponding stress tensor $\underline{S}_{\mathrm{vol}}$ and material tangent $\underline{\mathbb{E}}_{\mathrm{vol}}$ are computed by the first and second derivative with respect to the strain tensor $\underline{\underline{C}}_{\mathrm{vol}}$, respectively. For rubber-like materials, the change in volume is close to zero. Hence, the bulk modulus

$$
\kappa_{0}=\frac{4 C_{10}(1+v)}{3 \gamma_{\infty}(1-2 v)},
$$


where $C_{10}$ is the first YEOH parameter of the equilibrium branch and $v=0.49$ POISSON's ratio, is adjusted so that the material is almost incompressible. For this reason, only the material parameters of the isochoric part of the constitutive law (see Figure 4) need to be identified.

\subsubsection{Identification of Elastic Parameters}

By performing uniaxial, biaxial, and pure shear tests at so-called multi-step test conditions, the long-term response of the rubber compound analyzed in this work is investigated. The tests begin with a pre-conditioning step, where the Mullins effect, softening of the virgin rubber, is eliminated. Afterwards, the sample is relaxed up to a zero stress state and the multi-step test is carried out, which consists of loading to a certain strain level and holding of the strain level for a certain time. After the relaxation period has been reached, the rubber is loaded to a new strain level until the maximum strain in the multi-step test has been reached. During the unloading, the same routine of applying the strain and holding is carried out. By keeping the material at a strain level, it is assumed that all viscoelastic features of the rubber are eliminated and only the long-term behavior is active. For identification of the long-term response, only the fully relaxed stress state of each strain level is of interest. Therefore, a post-processor is detecting these points, which are highlighted in Figure 5 (exp. long-term). In the experiments, the shape of the specimens is chosen in a way to ensure a uniform deformation state. Hence, the deformation gradient can be expressed in principal direction as

$$
\underline{\boldsymbol{F}}=\left[\begin{array}{ccc}
\lambda_{1} & 0 & 0 \\
0 & \lambda_{2} & 0 \\
0 & 0 & \lambda_{3}
\end{array}\right] .
$$

The principal strains are computed as the ratio of the current length to the original length. In the case of rubber, incompressibility is assumed and, therefore, the relation

$$
J=\operatorname{det}(\underline{\boldsymbol{F}})=\lambda_{1} \lambda_{2} \lambda_{3}=1
$$

has to be fulfilled. Thus, $\lambda_{i}$ with $i=1,2,3$ are equal to the isochoric principal strains in Equation (17). For uniaxial cases, the first principle strain is measured as $\lambda_{1}=\lambda_{\mathrm{UT}}$ and according to Equation (21), the strains perpendicular to the loading direction are

$$
\lambda_{2}=\lambda_{3}=\frac{1}{\sqrt{\lambda_{\mathrm{UT}}}} .
$$

Consequently, the first invariant in uniaxial tension case is

$$
I_{\mathrm{UT}}=\lambda_{\mathrm{UT}}^{2}+\frac{2}{\lambda_{\mathrm{UT}}} \text {. }
$$

Biaxial tension tests are performed to include a more complex deformation mode in the parameter fitting procedure. In this case, the loading strains in the first and second direction are controlled, $\lambda_{1}=\lambda_{2}=\lambda_{\mathrm{BT}}$, and the remaining strain component is computed analogously to the uniaxial tension mode using the incompressibility condition Equation (21),

$$
\lambda_{3}=\frac{1}{\lambda_{\mathrm{BT}}^{2}} \text {. }
$$

The first invariant is then, accordingly,

$$
I_{\mathrm{BT}}=2 \lambda_{\mathrm{BT}}^{2}+\frac{1}{\lambda_{\mathrm{BT}}^{4}} .
$$

The last deformation mode investigated experimentally is a so-called pure shear test. For the pure shear case, the load is applied in direction of $\lambda_{1}$. To ensure plane strain state, the test sample has to be several orders larger in direction of $\lambda_{2}$ than in direction of $\lambda_{3}$. For this reason, the principle strains at consideration of incompressibility conditions are 


$$
\lambda_{1}=\lambda_{\mathrm{PS}}, \quad \lambda_{2}=1, \quad \lambda_{3}=\frac{1}{\lambda_{\mathrm{PS}}},
$$

and the first invariant is

$$
I_{\mathrm{PS}}=1+\lambda_{\mathrm{PS}}^{2}+\frac{1}{\lambda_{\mathrm{PS}}^{2}} .
$$

According to [17], the engineering stress can be computed by the chain rule

$$
\sigma_{\mathrm{eq}}=\frac{\partial \Psi_{\mathrm{eq}}}{\partial \lambda_{1}}=\frac{\partial \Psi_{\mathrm{eq}}}{\partial I} \frac{\partial I}{\partial \lambda_{1}} .
$$

Using the $\mathrm{YEOH}$ model as free energy density function leads to the following expression for the engineering stresses

$$
\sigma_{\mathrm{eq}}=\left[C_{10}+C_{20}(I-3)+C_{30}(I-3)^{2}\right] \frac{\partial I}{\partial \lambda_{1}} .
$$

For every experimental data point, the according numerical solution should be identical. The introduced equation for the numerical engineering stress (Equation (29)), is rewritten as

$$
\sigma_{\mathrm{eq}}=\frac{\partial I}{\partial \lambda_{1}}\left[\begin{array}{lll}
1 & (I-3) & (I-3)^{2}
\end{array}\right]\left[\begin{array}{l}
C_{10} \\
C_{20} \\
C_{30}
\end{array}\right] .
$$

For the identification of the unknown YEOH parameters, a linear system of equations (LSE)

$$
\underline{\boldsymbol{c}}_{\mathrm{eq}}\left[\begin{array}{l}
C_{10} \\
C_{20} \\
C_{30}
\end{array}\right]=r_{\mathrm{eq}}
$$

is built up with the coefficients of the right hand side vector $r_{\mathrm{eq}, i}=\sigma_{\mathrm{exp}, i}$ with $i=1, \ldots, m$ (number of experimental data points) and the coefficients

$$
c_{\mathrm{eq}, i}=\frac{\partial I_{i}}{\partial \lambda_{1, i}}\left[\begin{array}{lll}
1 & \left(I_{i}-3\right) & \left(I_{i}-3\right)^{2}
\end{array}\right]
$$

for row $i$ of the coefficient matrix. Solving Equation (31) results in the three material parameter $C_{10}, C_{20}$ and $C_{30}$. Figure 5 illustrates the fitted long-term response regarding (a) uniaxial tension, (b) biaxial tension, and (c) pure shear at $\vartheta=20^{\circ} \mathrm{C}$.

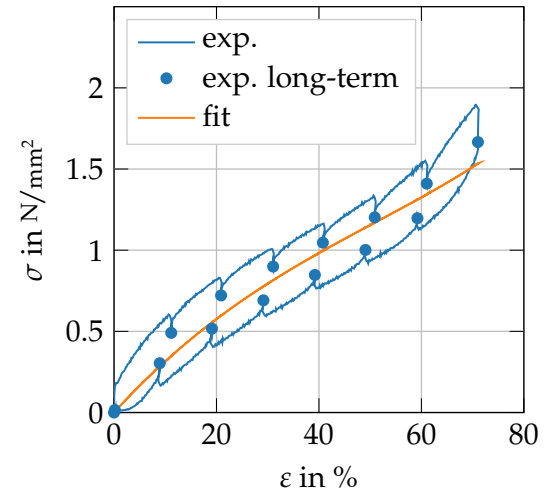

(a)

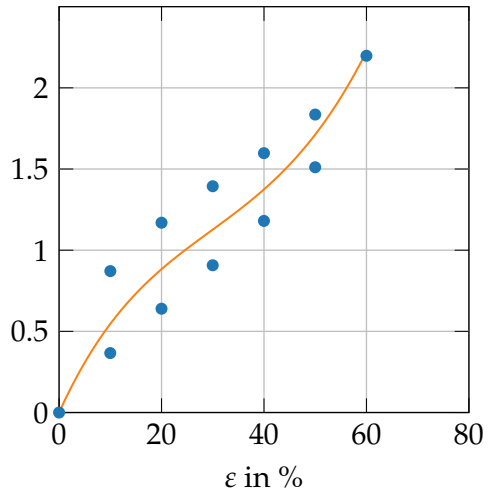

(b)

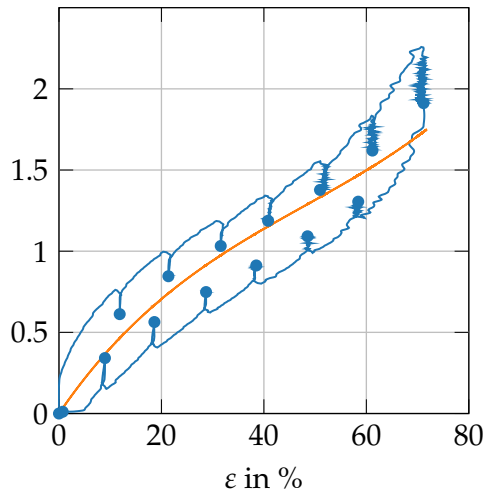

(c)

Figure 5. Fitting of long-term response of rubber compound at $\vartheta=20^{\circ} \mathrm{C}$. (a) Uniaxial tension, (b) Biaxial tension, (c) Pure shear.

The corresponding YEOH material parameters are listed in Table 1. 
Table 1. YEOH material parameters.

\begin{tabular}{ccc}
\hline$C_{\mathbf{1 0}}$ & $C_{\mathbf{2 0}}$ & $C_{\mathbf{3 0}}$ \\
\hline $0.57935 \mathrm{~N} / \mathrm{mm}^{2}$ & $-0.04953 \mathrm{~N} / \mathrm{mm}^{2}$ & $0.02483 \mathrm{~N} / \mathrm{mm}^{2}$ \\
\hline
\end{tabular}

\subsubsection{Identification of Viscoelastic Parameters}

For the identification of storage and loss moduli of the generalized MAXWELL model (see Equations (7) and (8)), the master curve (storage and loss moduli as function of applied frequency) of a dynamic mechanical analysis (DMA) is used. The temperature of the rubber sample is varied from -50 to $80^{\circ} \mathrm{C}$ during the DMA. At each temperature point, six different frequencies (load rate) from 0.5 to $30 \mathrm{~Hz}$ are investigated. By applying the WILLIAMS-LANDEL-FERRY (WLF) equation, a frequency range of $10^{-7}$ to $10^{9} \mathrm{~Hz}$ is generated at a reference temperature of $\vartheta=20^{\circ} \mathrm{C}$. For the identification of the viscoelastic material parameters, this frequency band is limited to $\left[10^{-3}, 10^{6}\right] \mathrm{Hz}$ (see Figure 6). First, the relaxation times

$$
\tau_{k}=\frac{1}{\min \left(\omega_{\exp }\right) 10^{[2(k-1)+1] s_{\log }}}
$$

with

$$
s_{\log }=\frac{\log \max \left(\boldsymbol{\omega}_{\exp }\right)-\log \min \left(\boldsymbol{\omega}_{\exp }\right)}{2 n}
$$

of all dashpots in Figure 4 are calculated by distributing them equally in the logarithmic space between the minimum and maximum applied frequency $f_{\text {exp }}$ during the experiment $\left(\omega_{\exp }=2 \pi f_{\text {exp }}\right)$. Two weighting factors $w^{\prime}$ and $w^{\prime \prime}$ are introduced to fit the normalized stiffness factors $\gamma=\left[\gamma_{\infty}, \gamma_{1}, \ldots, \gamma_{n}\right]^{T}$ to the storage and loss moduli of the experiments separately. For each modulus, a linear system of equations

$$
\underline{c}^{\square} \cdot \gamma^{\square}=r^{\square}, \quad \square \in\{l, \prime \prime\}
$$

is built up with the corresponding coefficient matrices

$$
c_{i j}^{\prime}= \begin{cases}E_{0} & \text { if } j=1 \\ \frac{\left(\omega_{i} \tau_{j-1}\right)^{2}}{1+\left(\omega_{i} \tau_{j-1}\right)^{2}} E_{0} & \text { otherwise }\end{cases}
$$

and

$$
c_{i j}^{\prime \prime}= \begin{cases}0 & \text { if } j=1 \\ \frac{\omega_{i} \tau_{j-1}}{1+\left(\omega_{i} \tau_{j-1}\right)^{2}} E_{0} & \text { otherwise }\end{cases}
$$

as well as the right hand side vectors $r_{i}^{\prime}=E_{\exp , \mathrm{i}}^{\prime}$ and $r_{i}^{\prime \prime}=E_{\mathrm{exp}, \mathrm{i}}^{\prime \prime}$ with $i=1, \ldots, m$ (number of experimental data points) and $j=1, \ldots, n+1$, where $m$ is the number of experimental data points and $n$ the number of MAXWELL elements. By solving Equation (35), the normalized stiffness factors $\gamma_{k}=w^{\prime} \gamma_{j}^{\prime}+w^{\prime \prime} \gamma_{j}^{\prime \prime}$ (with $k=\infty$ if $j=1$ and $k=j-1$ otherwise) are computed. In addition, the relaxation times $\tau_{k}$, obtained from Equation (33), are adapted in order to minimize the error between experimental and fitted storage and loss moduli

$$
\epsilon=\sqrt{\frac{\sum_{i=1}^{m}\left(E_{i}^{\prime} / E_{\exp , i}^{\prime}-1\right)^{2}+\left(E_{i}^{\prime \prime} / E_{\text {exp }, i}^{\prime \prime}-1\right)^{2}}{m}},
$$

where $E_{i}^{\prime}$ and $E_{i}^{\prime \prime}$ are computed by Equations (7) and (8) using the angular frequency from experiments $\omega_{\exp , i}=2 \pi f_{\exp , i}$, the fitted relaxation times $\tau_{k}$ and the normalized stiffness factors $\gamma_{k}$ resulting from Equation (35). If negative normalized stiffness factors result from solving Equation (35), the corresponding MAXWELL element is removed from the generalized MAXWELL model. 
Since the identification of the elastic $\left(C_{10}, C_{20}\right.$ and $\left.C_{30}\right)$ and the viscoelastic parameters $\left(\gamma_{k}\right.$ and $\tau_{k}$ ) is carried out independently, the long-term factor

$$
f_{\infty}=\frac{\gamma_{\infty} E_{0}}{4 C_{10}(1+v)}
$$

is required to ensure the same long-term response of both fitting procedures. Within a loop, the normalized stiffness factors $\gamma_{k}^{[l+1]}=f_{\infty}^{[l]} \gamma_{k}^{[l]}$ are updated and normalized again until $f_{\infty}^{[l]}$ is close to one. The results of the parameter identification (index fit) are shown in Figure 6.

The subscript 1 refers to the initial fitting of storage and loss moduli and the dotted lines (subscript 2) belong to the master curve applying the long-term factor. The weighting of the loss modulus within the fitting is chosen to be two times larger than the storage modulus $\left(w^{\prime}=1 / 3\right.$ and $\left.w^{\prime \prime}=2 / 3\right)$ to reduce the relative error for both contributions suitably. The number of applied MAXWELL elements is $n=15$. The resulting material parameters of the MAXWELL elements are listed in Table 2.

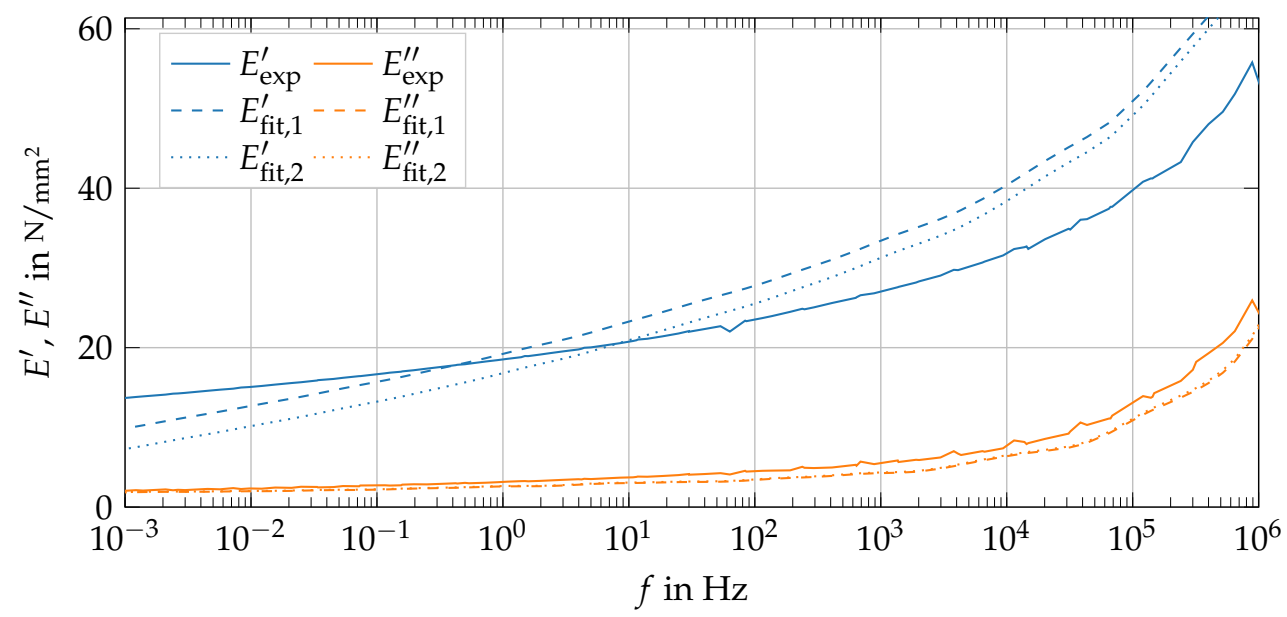

Figure 6. Master curve of rubber compound at $\vartheta=20^{\circ} \mathrm{C}$.

Table 2. Material parameters of the generalized MAXWELL model.

\begin{tabular}{ccc}
\hline Branch $k$ & $\gamma_{k}$ & $\tau_{k}$ \\
\hline 1 & 0.02301 & $8.01352 \times 10^{+2} \mathrm{~s}$ \\
2 & 0.01429 & $9.67013 \times 10^{+1} \mathrm{~s}$ \\
3 & 0.01326 & $1.97988 \times 10^{+1} \mathrm{~s}$ \\
4 & 0.01520 & $4.05365 \times 10^{+0} \mathrm{~s}$ \\
5 & 0.01613 & $7.76879 \times 10^{-1} \mathrm{~s}$ \\
6 & 0.01923 & $1.59060 \times 10^{-1} \mathrm{~s}$ \\
7 & 0.02194 & $2.67096 \times 10^{-2} \mathrm{~s}$ \\
8 & 0.02132 & $5.84217 \times 10^{-3} \mathrm{~s}$ \\
9 & 0.02575 & $1.04805 \times 10^{-3} \mathrm{~s}$ \\
10 & 0.03220 & $2.00858 \times 10^{-4} \mathrm{~s}$ \\
11 & 0.03083 & $3.15716 \times 10^{-5} \mathrm{~s}$ \\
12 & 0.04522 & $9.60953 \times 10^{-6} \mathrm{~s}$ \\
13 & 0.08282 & $1.23883 \times 10^{-6} \mathrm{~s}$ \\
14 & 0.06142 & $3.77065 \times 10^{-7} \mathrm{~s}$ \\
15 & 0.55373 & $3.73187 \times 10^{-8} \mathrm{~s}$ \\
\hline
\end{tabular}

The investigated frequency range $\left(f=10^{-3}\right.$ to $\left.10^{6} \mathrm{~Hz}\right)$ illustrated in Figure 6 covers the required frequency spectrum applied in the simulation. 


\subsection{Wear Model}

Wear is a consequence of friction between contact partners [18]. In this work, a combination of ARCHARD's wear model (see [19]) also known as REYE-ARCHARD-KHRUSHCHOV wear law and the abrasion law by SCHALLAMACH (see [20]) are applied. In ARCHARD's wear model, wear volume

$$
V_{w, \mathrm{RAK}}=\frac{k}{H} F_{N} L
$$

is a result of normal contact forces $F_{N}$, sliding distance $L$, material hardness $H$, and wear coefficient $k$. In contrast, SCHALLAMACH directly links the frictional energy (simplified as $\left.\mu F_{N} L\right)$ to the resulting wear volume

$$
V_{w, S}=\gamma \mu F_{N} L,
$$

where $\gamma$ represents the rate of abrasion per unit rate of energy dissipation. In recent publications (e.g., $[3,4,21])$, the abrasion rate law

$$
\dot{V}_{w}=k_{w} \cdot \dot{\mathscr{D}}_{s}^{a_{w}}
$$

with frictional dissipation rate or frictional energy rate $\dot{\mathscr{D}}_{s}$, wear coefficient $k_{w}$ and wear exponent $a_{w}$ is an extension of the approaches of ARCHARD and SCHALLAMACH. The wear model implemented in this work is based on Equation (42), where the frictional energy rate

$$
\dot{\mathscr{D}}_{\mathrm{S}}=\int_{A} \boldsymbol{t}_{T} \boldsymbol{v}_{T} d A
$$

is the integral of contact shear stresses $\boldsymbol{t}_{T}$ and sliding velocity vector $\boldsymbol{v}_{T}$ over the area of the contact surface $A$. The shear stresses

$$
\boldsymbol{t}_{T}=\mu p_{N} \varphi_{i} \frac{\boldsymbol{g}_{T}}{\left\|\boldsymbol{g}_{T}\right\|}
$$

are calculated by the coefficient of friction $\mu$, the normal pressure $p_{N} \geq 0$, the contact slip vector $\boldsymbol{g}_{T}$ and the function $\phi_{i}$, that describes the transition from sticking to sliding. In [22], an overview of different regularization functions, e.g.

$$
\varphi_{1}= \begin{cases}\frac{\left\|g_{T}\right\|}{g_{\text {crit }}} & \text { if }\left\|g_{T}\right\|<g_{\text {crit }} \\ 1 & \text { otherwise }\end{cases}
$$

or

$$
\varphi_{2}=\tanh \left(\frac{\left\|g_{T}\right\|}{\varepsilon}\right)
$$

are given. If the hyperbolic tangent regularization $\varphi_{2}$ (Equation (46)) is applied, the transition region (sticking to sliding) is differentiable, which is shown in Figure 7. 


\section{frictional shear stress $t_{T}$}

Coulomb's law

bilinear reg.

hyperbolic reg. $\left(\varepsilon=g_{\text {crit }}\right)$

hyperbolic reg. $\left(\varepsilon=0.5 g_{\text {crit }}\right)$
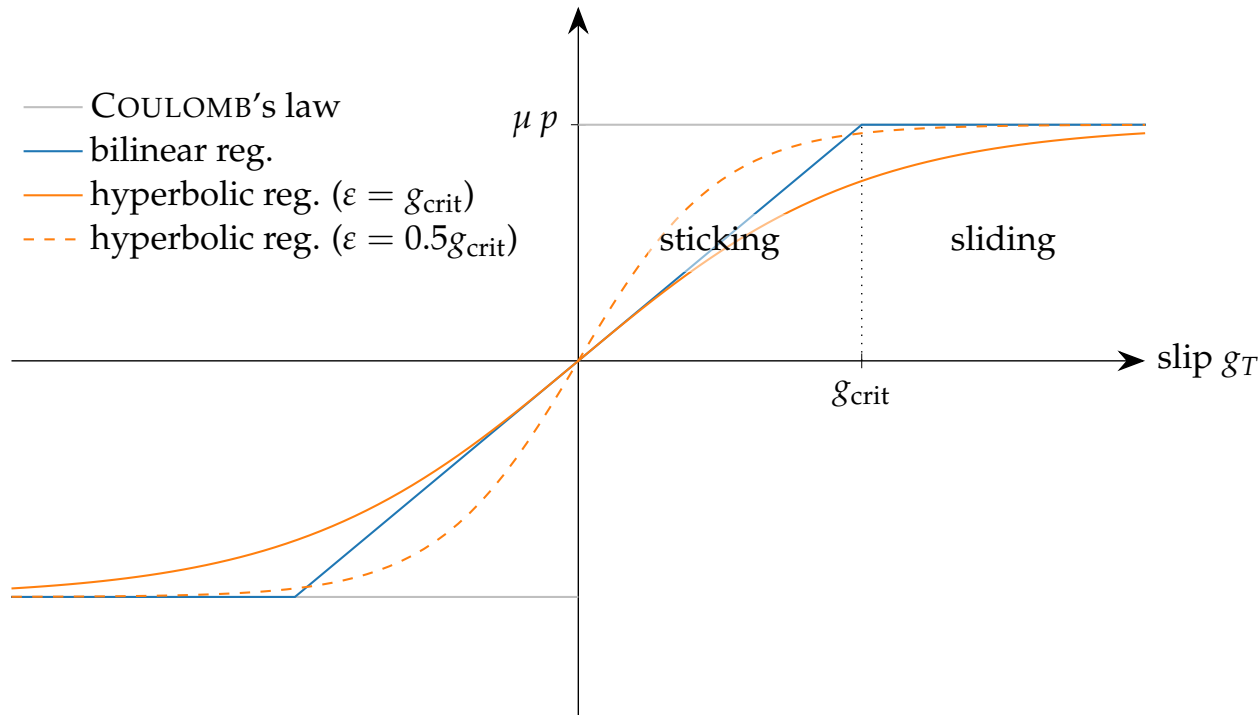

Figure 7. Regularization of COULOMB's friction law.

\subsubsection{Post-Processing Re-Meshing Algorithm}

In the following, the implementation of the wear model as well as the post-processing re-meshing algorithm are outlined. Figure 8 illustrates the schematic explanation for a simple two-dimensional finite element block with six elements sliding over a rough surface.

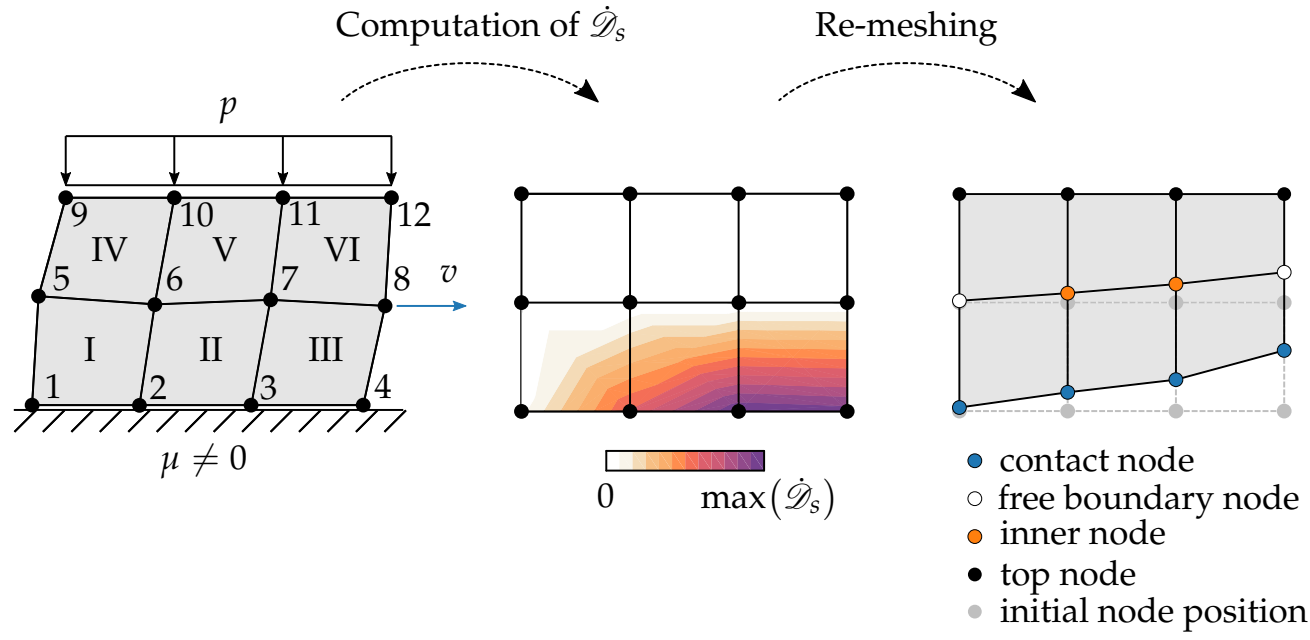

Figure 8. Schematic illustration of re-meshing algorithm.

The computation of the frictional energy

$$
\mathscr{D}_{s, i}\left(t_{n+1}\right)=\mathscr{D}_{s, i}\left(t_{n}\right)+\frac{\left\|\boldsymbol{F}_{T, i}\left(t_{n}\right)\right\|+\left\|\boldsymbol{F}_{T, i}\left(t_{n+1}\right)\right\|}{2}\left\|\Delta \boldsymbol{g}_{T, i}\left(t_{n+1}\right)\right\|
$$

of node $i$ and time step $t_{n+1}$ is performed as a post-processing operation after the finite element simulation has been completed. In Equation (47), $\boldsymbol{F}_{T, i}\left(t_{n+1}\right)$ is equal to the contact shear stresses integrated over its nodal area at time step $t_{n+1}$ and

$$
\Delta \boldsymbol{g}_{T, i}\left(t_{n+1}\right)=\left[\begin{array}{l}
g_{T, i, 1}\left(t_{n+1}\right)-g_{T, i, 1}\left(t_{n}\right) \\
g_{T, i, 2}\left(t_{n+1}\right)-g_{T, i, 2}\left(t_{n}\right)
\end{array}\right]
$$


relates to the change in contact slip between time step $t_{n}$ and $t_{n+1}$ of the same node. A contour plot of the frictional energy rate

$$
\dot{\mathscr{D}}_{s, i}\left(t_{n+1}\right)=\frac{\mathscr{D}_{s, i}\left(t_{n+1}\right)-\mathscr{D}_{s, i}\left(t_{n}\right)}{\Delta t}
$$

of the block model example is shown in the center image of Figure 8. In Equation (49), $\Delta t$ is defined by $t_{n+1}-t_{n}$. After the frictional energy rate computation has been completed, re-meshing starts. The re-meshing algorithm contains three procedures: moving contact nodes, free boundary nodes, and inner nodes (see right image in Figure 8). The wear height

$$
h_{w, i}\left(t_{n+1}\right)=h_{w, i}\left(t_{n}\right)+\frac{V_{w, i}\left(t_{n+1}\right)-V_{w, i}\left(t_{n}\right)}{A_{0, i}\left(t_{n+1}\right)}
$$

is computed cumulatively out of the incremental wear volume resulting from Equation (42) and the nodal area in the reference configuration (see explanation below). The new position of each contact node $\boldsymbol{x}_{\mathrm{w}, i}=\boldsymbol{X}_{\mathrm{w}, i}+\boldsymbol{u}_{\mathrm{w}, i}$ is defined by

$$
\boldsymbol{u}_{w, i}=\sum_{n=1}^{n_{t}-1}-\left[h_{w, i}\left(t_{n+1}\right)-h_{w, i}\left(t_{n}\right)\right] \overline{\boldsymbol{n}}_{i}\left(t_{n+1}\right)
$$

where $n_{t}$ coincides with the number of time steps and $\bar{n}_{i}$ is the unit-length normal vector $\left(\left\|\overline{\boldsymbol{n}}_{i}\right\|=1\right)$ of node $i$ in the current configuration. $\boldsymbol{n}_{i}$ is obtained by averaging the normal vectors of all element faces that are connected to node $i$ and belong to the contact surface. To apply the abrasion vector to the nodal coordinates in the reference configuration, $\boldsymbol{u}_{w, i}$ should be left multiplied by the inverse of the deformation gradient at node $i$, if the nodal area in the current configuration is used in Equation (50). However, the deformation gradient is only defined at the integration points within the element. A projection to the element nodes by shape functions increases the computational costs significantly and leads to an unknown error due to averaging at nodes, which are connected to multiple elements. For this reason, the nodal area in the reference configuration is considered instead in Equation (50). This assumption is verified in Section 3.3. Since the computation of the wear height in Equation (50) is approximated by using the nodal area, an internal iteration minimizes the difference between the node based wear volume $V_{w, i}$ and the resulting wear volume on the element level, taking the new position of the contact nodes into account. For this purpose, each displacement vector $\boldsymbol{u}_{w, i}$ is multiplied by an abrasion factor $f_{w, i}$ with $f_{w, i}^{[1]}=1$ for the first iteration. By comparing nodal and elemental wear, this factor varies in the range of $1 \pm f_{w, \max }$ with $f_{w \text {, } \max }<1$ for each node separately. After the new position of the contact nodes (blue circles in Figure 8 on the right) is known, the boundary nodes, which belong neither to the top nor the contact nodes, are moved as follows. First, the possible moving directions (only along element edges) of each free boundary node $i$ (white circles in Figure 8 on the right), which is attached to a contact node $j$, are identified. The decisive direction is detected by computing the dot products

$$
d_{\mathrm{fb}, k}=\left(\boldsymbol{X}_{\mathrm{fb}, i}-\boldsymbol{X}_{w, j}\right) \cdot\left(\boldsymbol{X}_{k}-\boldsymbol{X}_{\mathrm{fb}, i}\right)
$$

of vector $\boldsymbol{X}_{\mathrm{fb}, i}-\boldsymbol{X}_{w, j}$ (contact node $j$ to free boundary node $i$ ) and vector $\boldsymbol{X}_{k}-\boldsymbol{X}_{\mathrm{fb}, i}$ (free boundary node $i$ to connected element edge node $k$ ) with $k \neq j$. Node $k=\operatorname{argmax} d_{\mathrm{fb}}$ defines the resulting moving direction of free boundary node $i$, which is moved by

$$
\boldsymbol{u}_{\mathrm{fb}, i}=\frac{\left\|\boldsymbol{u}_{w, j}\right\| \frac{d_{\mathrm{fb}, ~}}{\Delta X_{\mathrm{fb}, i}}}{\Delta X_{\mathrm{fb}, i}+\frac{d_{\mathrm{fb}, k}}{\Delta X_{\mathrm{fb}, i}}} \frac{\boldsymbol{X}_{k}-\boldsymbol{X}_{\mathrm{fb}, i}}{\left\|\boldsymbol{X}_{k}-\boldsymbol{X}_{\mathrm{fb}, i}\right\|}
$$

with $\Delta X_{\mathrm{fb}, i}=\left\|X_{\mathrm{fb}, i}-X_{w, j}\right\|$ to get its new position $x_{\mathrm{fb}, i}=X_{\mathrm{fb}, i}+\boldsymbol{u}_{\mathrm{fb}, i}$. Subsequently, the next row of free boundary nodes, which are connected to the previously modified 
boundary nodes, are moved analogously by replacing the subscript index $\square_{w, j}$ to $\square_{f b, j}$ in Equations (52) and (53) (not relevant for block model in Figure 8). Finally, the location of the inner nodes (orange circles in Figure 8 on the right) is updated by using the condition

$$
f_{\mathrm{in}, i}=\sum_{j=1}^{n_{\mathrm{el}}} \alpha\left(x_{\mathrm{el}, j}-x_{\mathrm{in}, i}\right)=\mathbf{0}
$$

for each inner node $i$ with $n_{\mathrm{el}}$ (number of elements), the center coordinates $x_{\mathrm{el}, j}$ of element $j$ and $\alpha=1$ if node $i$ is an element node of element $j$ (zero otherwise). If this condition is fulfilled, each inner node lies in the center of a virtually created element using the center of its attached elements as element nodes. From Equation (54), a linear system of equations

$$
\underline{\boldsymbol{K}}_{\mathrm{in}} \cdot \boldsymbol{x}_{\mathrm{in}}=\boldsymbol{r}_{\mathrm{out}}
$$

is generated for all inner nodes in each dimension. The coefficient matrix

$$
\underline{K}_{\text {in }}=\frac{\partial f_{\text {in }}}{\partial x_{\text {in }}}
$$

results from the derivative of Equation (54) with respect to the coordinates of the inner nodes. Contributions in Equation (54), that belong to the outer nodes (top nodes, contact nodes and free boundary nodes), are shifted to the right hand side of the LSE to form $r_{\text {out }}$ In the two-dimensional case (like in Figure 8), two LSE are solved for the horizontal and the vertical coordinates respectively in order to find the new location of the inner nodes $x_{\text {in }}$ after re-meshing. The node coordinates of the top surface nodes (black circles in Figure 8 on the right) are fixed during re-meshing.

\subsubsection{Adaptive Meshing Algorithm}

In contrast to the post-processing re-meshing, the adaptive meshing algorithm computes the frictional energy rate

$$
\dot{\mathscr{D}}_{s, i}\left(t_{n+1}\right)=\left\|\boldsymbol{\tau}_{t, i}\left(t_{n+1}\right)\right\| A_{i}\left(t_{n+1}\right) \frac{\left\|\Delta \boldsymbol{g}_{T, i}\left(t_{n+1}\right)\right\|}{\Delta t}
$$

for each contact node $i$ right after the finite element solver completes the current time step. In Equation (57), $A_{i}$ is the current nodal area of node $i$, which is simplified as a rectangle in the three-dimensional space, where the length and width are half the distance to the adjacent nodes. In the two-dimensional case, the nodal area is represented by a line. The mesh motion

$$
h_{w, i}=\frac{\dot{V}_{w, i}\left(t_{n+1}\right) \Delta t}{A_{0, i}\left(t_{n+1}\right)}
$$

results from the incremental volume loss rate $\dot{V}_{w, i}$ (see Equation (42)), the time increment $\Delta t$ and the nodal area $A_{0, i}$ in the reference configuration. The abrasion direction always points against the surface normal of node $i$ in the current configuration. After the coordinates of all contact nodes are updated, the next time step starts. The wear model is implemented in the adaptive meshing algorithm of a commercial FE code using a user subroutine to specify mesh motion constraints during adaptive meshing. As described for the post-processing re-meshing algorithm, the application of the nodal area in the reference configuration in Equation (58) is preferred to the projection of the deformation gradient at the corresponding node position. Furthermore, access to the deformation gradient at the associated integration points is not given in the applied user subroutine. Again, this assumption is verified in Section 3.3. The computational efficiency of the adaptive meshing algorithm depends on the update frequency of the finite element mesh. If the block mesh is updated only once during the simulation, the adaptive meshing algorithm approaches the post-processing re-meshing algorithm. 


\subsection{Image Processing and Mesh Generation}

2.5.1. Image Processing

The profiles of the rubber samples are recorded by a camera after the run-in phase (pre-conditioning) and after the friction/wear test (see Section 2.1). These photographs of the rubber profiles are used to identify the boundary shape of the pre-conditioned as well as the worn rubber blocks. An image processing procedure is developed by applying different modules of the scikit-image package in Python [23]. In Figure 9, the work flow is illustrated exemplary for the pre-conditioned block at $p=1.2 \mathrm{~N} / \mathrm{mm}^{2}$ and $v=100 \mathrm{~mm} / \mathrm{s}$.

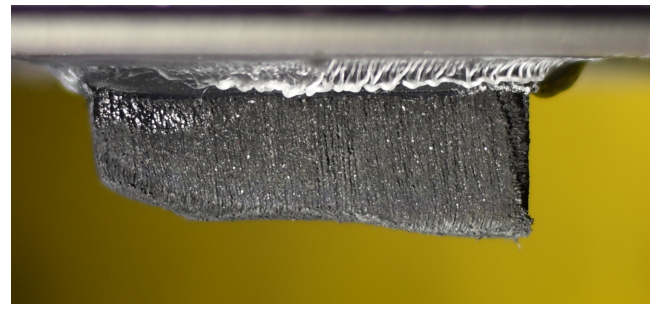

(a)

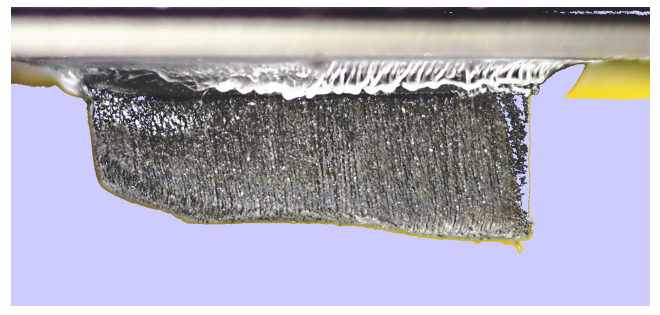

(c)

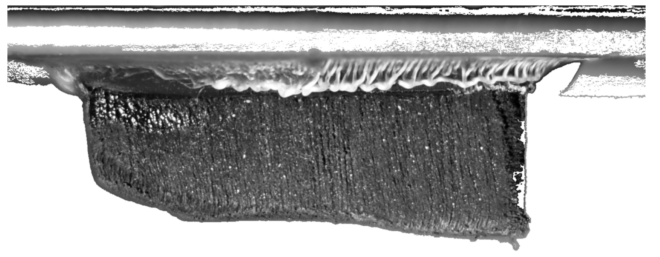

(e)

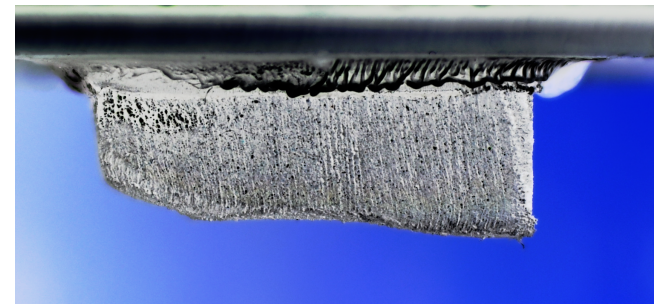

(b)

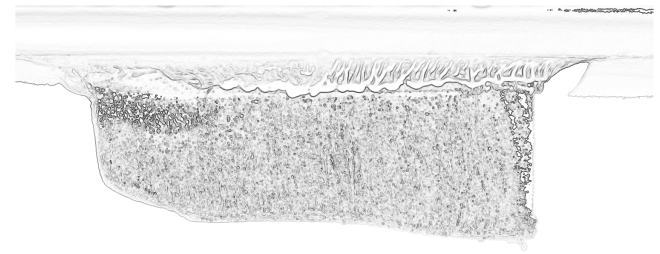

(d)

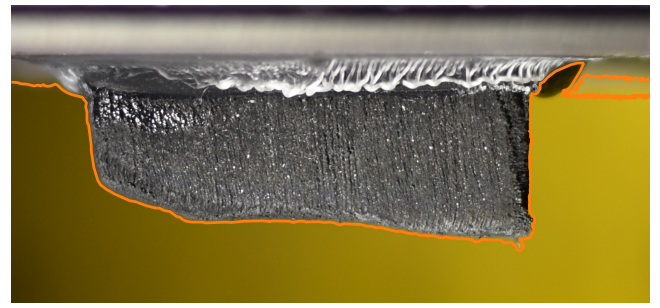

(f)

Figure 9. Image processing at $p=1.2 \mathrm{~N} / \mathrm{mm}^{2}$ and $v=100 \mathrm{~mm} / \mathrm{s}$. (a) Original, (b) Color inversion, (c) Background deletion, (d) SOBEL filter, (e) GAUSSian filter, (f) Contour identification.

First, the contrast of the image is adjusted by histogram equalization (see [24]) to improve the output during the color space inversion in Figure 9b. As result, the dark yellowish background in Figure 9a changes to different shades of blue. In the next step, the background can be removed by defining a minimal blue contribution from the red-greenblue (RGB) color model. In the digital RGB color model, each color channel is assigned a value between 0 and 255. Applying a normalized minimal blue contribution of 0.85 $(0.85 \times 255 \approx 217)$ leads to the outcome presented in Figure 9c. In the following step, the SOBEL filter (also called SOBEL-FELDMAN operator) is applied in order to detect the block edges [25]. For a suitable presentation in Figure 9d, the output of the SOBEL filter is visualized in the inverted gray-scale. Furthermore, a flood tool is used to identify connected points within a tolerance of the seed value starting from a specific seed point. To apply a multidimensional GAUSSian filter, which enhances the gray gradients at the edges, the original photo is transferred into gray scale. The color white is assigned to the connected points from the flood tool. Figure 9e shows the results using a tolerance value for the flood tool of 0.01 and a standard deviation for the GAUSsian kernel of 0.9. Finally, the block contour is identified 
by the marching-squares method to compute an iso-valued contour of a two-dimensional array [26]. The block contour for a level value of $0.9(0 \rightarrow$ black, $1 \rightarrow$ white).

To compare the recorded rubber profiles after the run-in phase and at the end of the linear friction test, the images are aligned. Initially, the ORB (Oriented FAST and Rotated BRIEF) keypoints detector is applied to identify similar points in both photos [27]. This procedure works for gray-scale images. The keypoints are illustrated by circles in Figure 10.

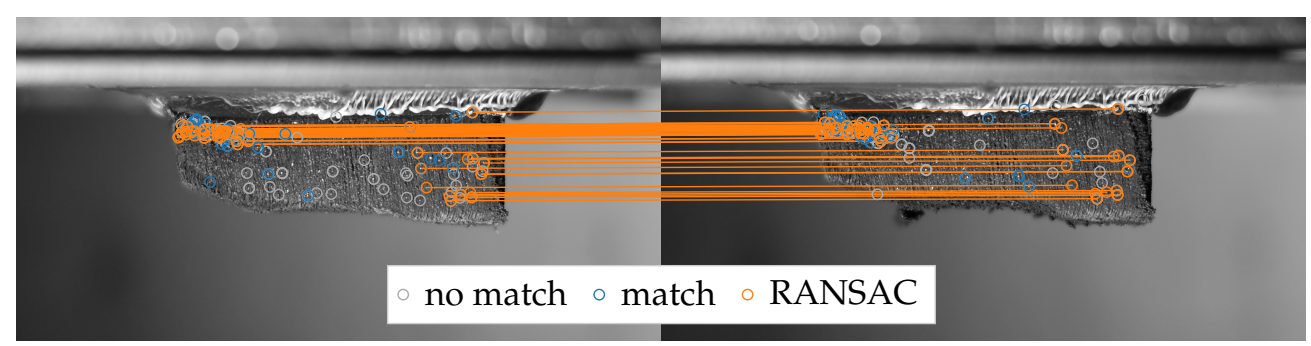

Figure 10. Image alignment at $p=\mathrm{N} / \mathrm{mm}^{2}$ and $v=100 \mathrm{~mm} / \mathrm{s}$.

Each keypoint has its own descriptor that characterizes the surrounding region. As the second step, the ORB detector finds matching descriptors from the first image in the second one (and vice-versa). These matching points are represented by blue circles in Figure 10. To improve the output of the ORB detector, a random sample consensus (RANSAC) is applied on top of the matching points [28]. The remaining connections of the alignment points are shown as orange lines in Figure 10. Finally, the second image (after the wear test) is warped according to a given coordinate transformation. The modified photos are used to evaluate wear along the contact surface of the rubber sample.

\subsubsection{Mesh Generation}

In order to scale the rubber block contour, the block coordinates with the largest horizontal distance to each other are selected manually (see the two black circles in Figure 11a). With the selected image coordinates and the measured rubber block length $l$, it is possible to transfer the image contour into the Cartesian coordinate system. The vertical location of the top surface of the rubber block is also user-defined, because the image processing procedure is not able to detect this surface correctly due to the layer of glue between rubber and mount. The modified contour is shown in Figure 11a as orange solid line. After identifying the contour from the photograph, the data points are smoothed by B-splines (see e.g., [29]). The smoothing condition is balanced between avoiding sharp asperities to ensure proper meshing and preserving the original contour as well as possible. In Figure 11a, the smoothed contour is illustrated by a green solid line. 


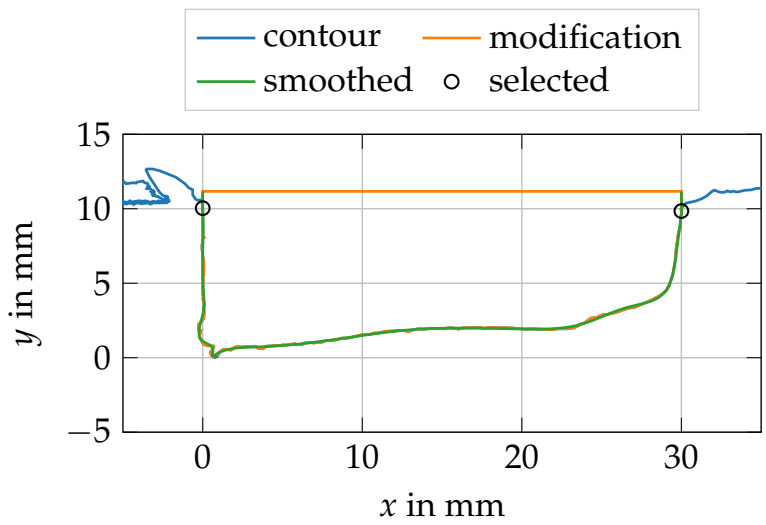

(a)

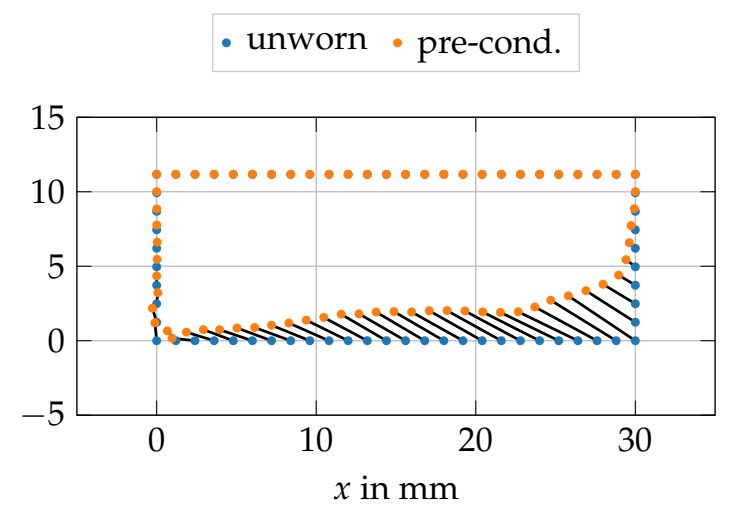

(b)

Figure 11. Contour scaling and edge seed at $p=0.8 \mathrm{~N} / \mathrm{mm}^{2}$ and $v=10 \mathrm{~mm} / \mathrm{s}$. (a) Contour scaling, (b) Edge seed.

To apply the finite element method (FEM), the rubber block is discretized by a mesh generator from a commercial FE code using the seed of the block contour (edge seed). The edge seeds of the unworn and pre-conditioned rubber blocks are illustrated in Figure 11b using equidistant nodes $(d=l / 25)$.

Different mesh configurations are analyzed in a two-dimensional as well as in threedimensional space. In Figure 12, three different two-dimensional FE block models are shown, which correspond to the recorded sample profile during experiment at $p=1.2 \mathrm{~N} / \mathrm{mm}^{2}$ and $v=100 \mathrm{~mm} / \mathrm{s}$. The length of the this rubber sample is $l=30 \mathrm{~mm}$ and its initial height is $h=11.245 \mathrm{~mm}$.

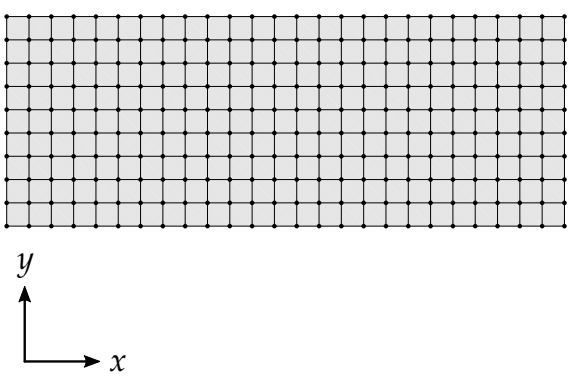

(a)

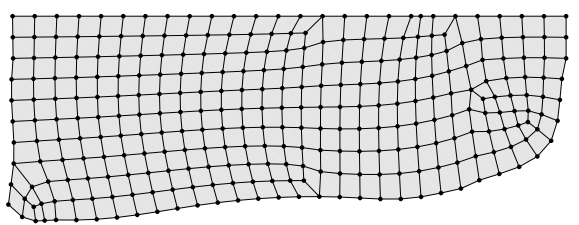

(b)

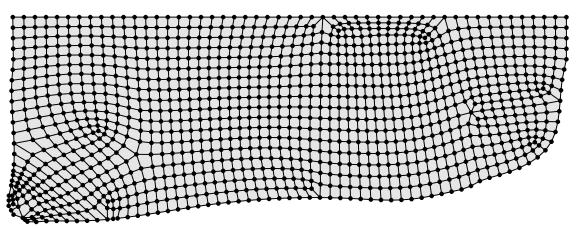

(c)

Figure 12. Two-dimensional FEM block models at $p=1.2 \mathrm{~N} / \mathrm{mm}^{2}$ and $v=100 \mathrm{~mm} / \mathrm{s}$. (a) 225 elements, unworn, (b) 256 elements, pre-cond, (c) 1026 elements, pre-cond.

Figure 12a illustrates the initial or unworn rubber block with $25 \times 9$ plain-strain elements (4-node bilinear). By contrast, the pre-conditioned block models in Figure 12b,c consist of 256 and 1026 elements (identical element type as in unworn block model), respectively. To generate three-dimensional block models, the two-dimensional models are extruded in the third dimension while renaming the vertical axis $(y \rightarrow z)$. The resultant 
block models at $p=1.2 \mathrm{~N} / \mathrm{mm}^{2}$ and $v=100 \mathrm{~mm} / \mathrm{s}$ are present in Figures 13, where the rubber sample width is $w=20 \mathrm{~mm}$.

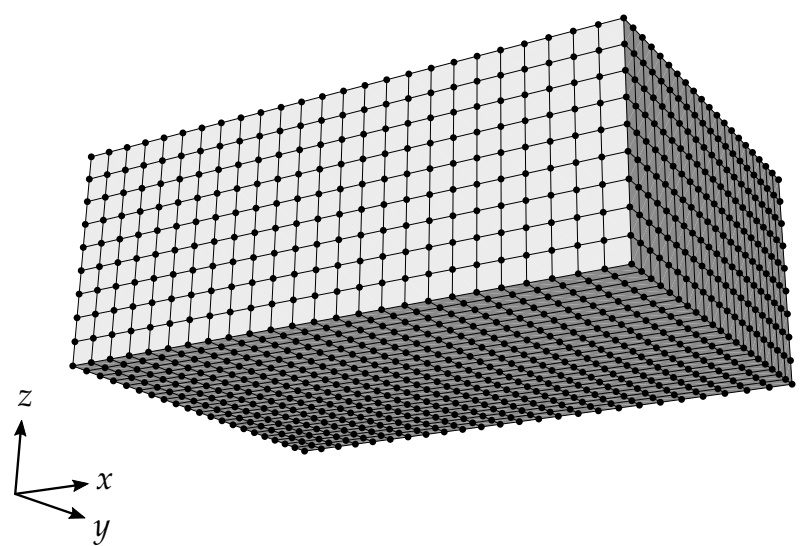

(a)

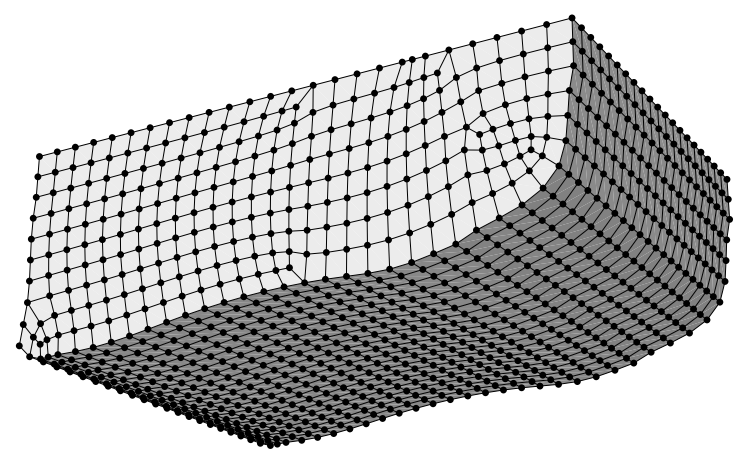

(b)

Figure 13. Three-dimensional FEM block models at $p=1.2 \mathrm{~N} / \mathrm{mm}^{2}$ and $v=100 \mathrm{~mm} / \mathrm{s}$. (a) 4500 elements, unworn, (b) 5120 elements, pre-cond.

The unworn block model in Figure 13a and the block model with a pre-conditioned shape (see Figure 13b) consist of $25 \times 9 \times 20$ elements and $256 \times 20$ elements, accordingly. Both block models apply three-dimensional 8-node linear brick elements.

\section{Results and Discussion}

\subsection{Experimental Output}

Figure 14a shows quasi-steady friction tests and Figure 14b illustrates the quasi-steady wear characteristics. All results were acquired according to procedures stated in Section 2.1 and represent mean values of five repetitions.

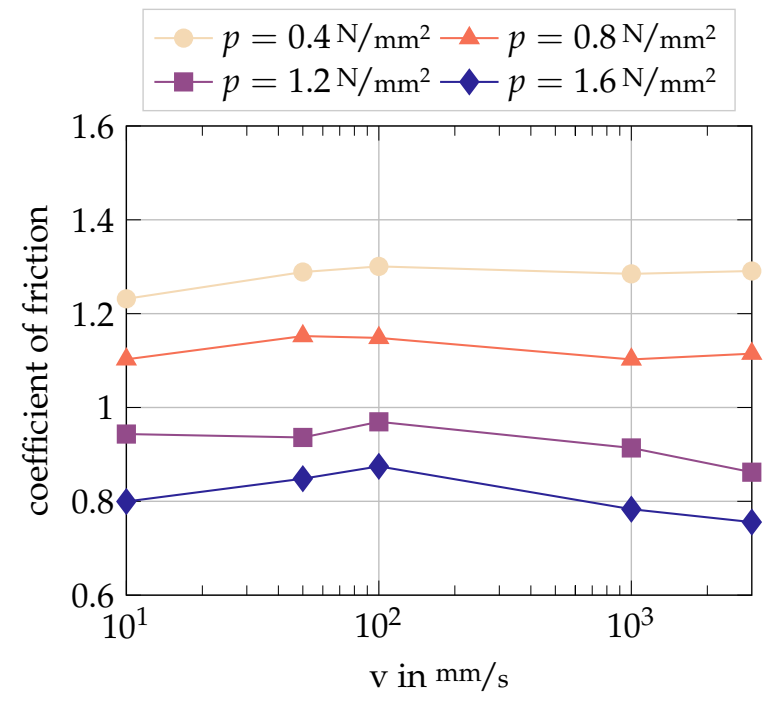

(a)

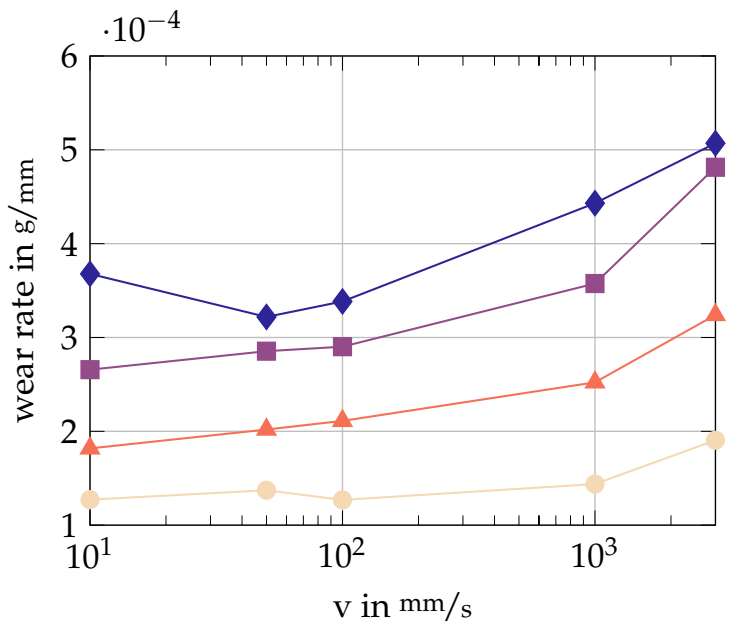

(b)

Figure 14. Output of linear friction and wear tests. (a) Coefficient of friction, (b) Wear rate.

The coefficient of friction, which is calculated in each measured time step from signals of normal force and tangential force sensors. The quasi-steady measurements are performed for 5 sliding velocities $(10,50,100,1000,3000 \mathrm{~mm} / \mathrm{s})$ and 4 contact pressure levels $(0.4$, $0.8,1.2,1.6 \mathrm{~N} / \mathrm{mm}^{2}$ ). This domain covers the relevant parameter range for the local tire footprint contact representing longitudinal dynamics of truck tires including ABS-braking 
and acceleration. The coefficient of friction of the truck compound on sand paper is in the range of 0.7 and 1.3, with a clearly decreasing trend over increasing contact pressure. This expected characteristics is a direct consequence of the real contact area of a compliant material on a solid rough surface. Above a certain contact pressure, the contact area cannot grow significantly, thus, the normal force is increasing but the transmissible tangential force is not. Hence, the coefficient of friction decreases. In terms of sliding velocity, this compound shows a relatively uniform friction level for each contact pressure stage.

The wear characteristics exhibit an increasing wear rate over both sliding velocity and contact pressure. This shows the close relation between wear rate and frictional power in the parameter range tested in the scope of this paper. The minimum at $50 \mathrm{~mm} / \mathrm{s}$ for high pressure characteristics is not considered as statistically significant here. The high absolute level of wear $(0.01$ to $0.05 \mathrm{mg} / \mathrm{mm}$ ) can be attributed to the strong abrasiveness of the sand paper track and the strict surface cleaning routine. The monotonous properties of the wear characteristics without maxima underline the assumption that only one wear mechanism is dominant (see [5]).

\subsection{Wear Parameter Identification}

The wear parameters introduced in Equation (42) (see Section 2.4) are identified by using the experimental output of the block tests described in Section 3.1. The frictional energy rate in the experiment

$$
\dot{\mathscr{D}}_{s, \exp }=\frac{\mu p_{N} A L}{\Delta t}
$$

considers the recorded friction coefficient $\mu$, the normal pressure $p_{N}$ applied on the top side of the block (area $A$ ), the sliding distance $L$ and the time period $\Delta t$ of each test. The abrasion rate in terms of volume

$$
\dot{V}_{w, \exp }=\frac{\dot{m}_{w}}{\rho}
$$

is computed by the mass loss rate (recorded mass difference divided by $\Delta t$ ) and the density of the applied rubber material $\rho=1.12 \times 10^{-3} \mathrm{~g} / \mathrm{mm}^{3}$.

To compare the abrasion rate from experiment and simulation, the averaged EUCLIDean distance

$$
\overline{\mathfrak{D}}_{2, \mathrm{abs}}=\sqrt{\frac{1}{n} \sum_{i=1}^{n}\left(\dot{V}_{w, \exp , i}-\dot{V}_{w, i}\right)^{2}}
$$

is applied to calculate the mean absolute error between both quantities. In Equation (61), $n$ represents the number of experimental data points. To obtain the relative error $\overline{\mathfrak{D}}_{2 \text {,rel }}$, the term $\left(\dot{V}_{w, \text { exp }, i}-\dot{V}_{w, i}\right)^{2}$ in Equation (61) is divided by $\dot{V}_{w, \text { exp }, i}^{2}$. An optimization tool identifies the wear parameters $k_{w}$ and $a_{w}$ by minimizing the error value defined in Equation (61). Figure 15 shows the result of the fitting procedure in logarithmic space.

A linear as well as a non-linear correlation between frictional energy rate and abrasion rate are investigated. Table 3 lists all parameter combinations including the resulting coefficient of determination

$$
R^{2}=1-\frac{\sum_{i=1}^{m}\left(\dot{V}_{w, \text { exp }, i}-\dot{V}_{w, i}\right)^{2}}{\sum_{i=1}^{m}\left(\dot{V}_{w, \text { exp }, i}-\bar{V}_{w, \exp }\right)^{2}},
$$

which indicate if the abrasion rate is predictable by the underlying wear model function. 


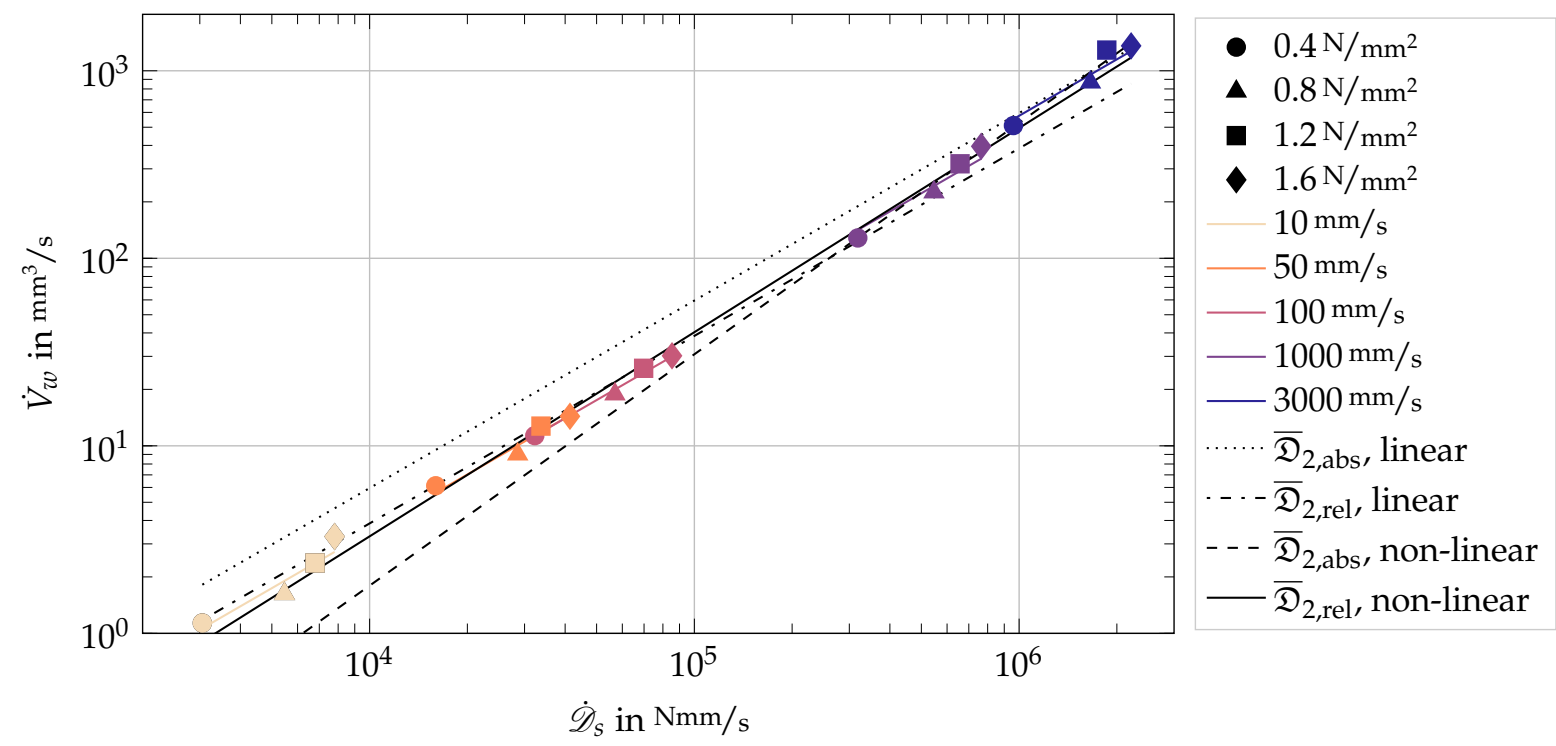

Figure 15. Wear parameter identification.

Table 3. Wear parameters and associated coefficient of determination.

\begin{tabular}{ccccc}
\hline Parameter & Linear (abs. Error) & Linear (rel. Error) & Non-Linear (abs. Error) & Non-Linear (rel. Error) \\
\hline$k_{w}$ in $\mathrm{mm}^{2} / \mathrm{N}$ & $5.951 \times 10^{-4}$ & $3.857 \times 10^{-4}$ & $2.083 \times 10^{-5}$ & $1.452 \times 10^{-4}$ \\
$a_{w}$ & 1 & 1 & 1.234 & 1.089 \\
\hline$R^{2}$ & 0.978 & 0.808 & 0.988 & 0.961 \\
\hline
\end{tabular}

By using the relative error $\overline{\mathfrak{D}}_{2, \text { rel }}$, the wear model covers the entire range sliding velocities of the experiments, especially the low frictional energy rates at low sliding velocities. The corresponding coefficients of determination (see Table 3, Columns 3 and 5 ) are lower in comparison to the $R^{2}$ values using the absolute error $\overline{\mathfrak{D}}_{2, \text { abs }}$, because Equation (62) does not consider relative differences. The wear parameters in Table 3, Columns 3 and 5 are applied for the validation of the wear model in Section 3.3.

\subsection{Wear Simulation and Comparison to Experiments \\ 3.3.1. Wear Simulation}

To simulate the experiments introduced in Section 2.1 in a proper manner, the generated FE block models described in Section 2.5 are applied within the different re-meshing algorithms (see Section 2.4) using the wear model parameters in Table 3. The counter surface as well as the mount of the rubber sample are modeled as rigid bodies (see Figure 16). Previously performed simulations have shown that the application of the mount is required to avoid unrealistic folding at top of the trailing edge of the rubber mesh during the sliding step.

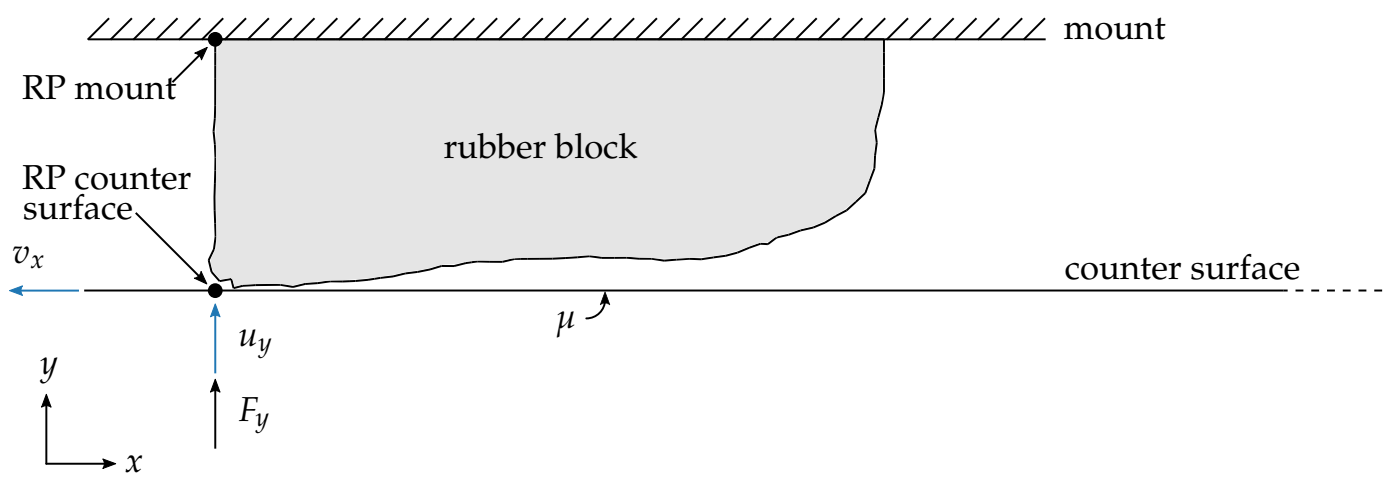

Figure 16. FE model setup. 
The augmented LAGRANGE method is used to enforce the contact constraints between the rubber block model and the rigid body surfaces [30]. Frictionless contact is assumed between the mount and the rubber block. The friction coefficient at the counter surface is changing during the simulation depending on the current step in order to model both surfaces aluminum and sandpaper. Table 4 gives an overview of the simulation steps for each test run.

Table 4. Steps of the FEM simulation.

\begin{tabular}{|c|c|c|c|}
\hline Number & Name & Description & $\begin{array}{l}\text { Boundary } \\
\text { Conditions }\end{array}$ \\
\hline 1 & Contact & $\begin{array}{l}\text { Counter surface is lifted to ensure contact } \\
\text { between rubber block and bottom rigid } \\
\text { surface }\end{array}$ & $\begin{array}{l}\Delta t=10^{-2} \mathrm{~s} \\
u_{v}=1 \mathrm{~mm}\end{array}$ \\
\hline 2 & Loading & $\begin{array}{l}\text { Counter surface is pressed against rubber } \\
\text { block }\end{array}$ & $\begin{array}{l}\Delta t=1 \mathrm{~s} \\
F_{v}=p_{N} A\end{array}$ \\
\hline 3 & Ramping & $\begin{array}{l}\text { Counter surface is accelerated while friction } \\
\text { is continuously increased }\end{array}$ & $\begin{array}{l}\Delta t=\frac{v}{a} \\
v_{x}=a t \\
\mu=\mu_{\mathrm{alu}} t\end{array}$ \\
\hline 4 & $\begin{array}{l}\text { Sliding-on- } \\
\text { aluminum }\end{array}$ & $\begin{array}{l}\text { Counter surface is moving with constant } \\
\text { speed and aluminum based friction }\end{array}$ & $\begin{array}{l}\Delta t=\frac{L_{\mathrm{alu}}-\frac{v^{2}}{2 a}}{v} \\
v_{x}=v \\
\mu=\mu_{\mathrm{alu}}\end{array}$ \\
\hline 5 & $\begin{array}{l}\text { Sliding-on- } \\
\text { sandpaper }\end{array}$ & $\begin{array}{l}\text { Counter surface is moving with constant } \\
\text { speed and sandpaper based friction }\end{array}$ & $\begin{array}{l}\Delta t=\frac{L_{\mathrm{sp}}}{v} \\
v_{x}=v \\
\mu=\mu_{\mathrm{sp}}\end{array}$ \\
\hline
\end{tabular}

Due to the glued connection between the mount and the top surface of the rubber sample in the experiment, all rubber block nodes located at the top surface are fixed during the entire simulation. To improve the convergence of the simulation, the counter surface is lifted vertically by $u_{v}=1 \mathrm{~mm}$ to get into contact with the rubber block during the first step. In the next step, the load $F_{v}=p_{N} A$, where $p_{N}$ is the normal pressure (applied in experiment) and $A$ represents the area of the top surface of the rubber block, is applied in vertical direction (positive $y$ - and $z$-direction in $2 \mathrm{D}$ and $3 \mathrm{D}$, respectively) at the reference point of the counter surface. In the two-dimensional case, $A$ is equal to the length of the block's top surface. In Step 3, the counter surface is accelerated by $a=5 \mathrm{~g}=49.05 \mathrm{~m} / \mathrm{s}^{2}$ horizontally (negative $x$-direction) and an averaged friction coefficient for aluminum recorded during the experiment is increased linearly. Subsequently, the counter surface is moved constantly by the predefined velocity $v$. The sliding distance on the aluminum sheet $L_{\text {alu }}$ is determined in the experiment (see Section 2.1). The friction property is changing to the averaged coefficient of friction, which is observed on sandpaper instantaneously at the beginning of the last step. The sliding distance $L_{\mathrm{sp}}$ corresponds to the elapsed sliding distance on the linear friction tester within one run.

Due to friction, the substrate as well as the rubber block is warmed-up. Since the coefficient of friction and the wear model are based on experimental data, friction as well as wear features due to temperature change are considered in the simulation. Heating of the rubber block during sliding is initially neglected in the simulation, because on average (of all laboratory tests), the temperature at the bottom surface of the rubber block has been changed by approximately $\Delta \vartheta=12.2^{\circ} \mathrm{C}$. The maximum temperature increment, which is recorded by the HiLiTe at $p=1.6 \mathrm{~N} / \mathrm{mm}^{2}$ and $v=3000 \mathrm{~mm} / \mathrm{s}$, is $\Delta \vartheta=27.1^{\circ} \mathrm{C}$. The influence of the temperature is part of future research using, e.g., a thermo-mechanically coupled block simulation and temperature-dependent parameters for the rubber material. After each test, the rubber sample is cooled down to ambient temperature $\left(\vartheta=20^{\circ} \mathrm{C}\right)$. 
If the post-processing re-meshing algorithm introduced in Section 2.4 is applied, the frictional energy rate is computed at each contact node of the rubber block after completion of the simulation. As soon as new node coordinates are computed by the re-meshing algorithm depending on the underlying wear model (linear or non-linear), the next test run with the same program listed in Table 4 starts. If the adaptive meshing algorithm is used, the new coordinates of the contact nodes are already known at the end of the simulation. Accordingly, only the location of the inner nodes and the free boundary nodes (see Figure 8 ) are updated by the re-meshing algorithm.

The comparison of the contact pressure distribution at $p=1.2 \mathrm{~N} / \mathrm{mm}^{2}$ and $v=100 \mathrm{~mm} / \mathrm{s}$ in Figure 17 demonstrates the influence of the applied block model shape as the initial configuration.
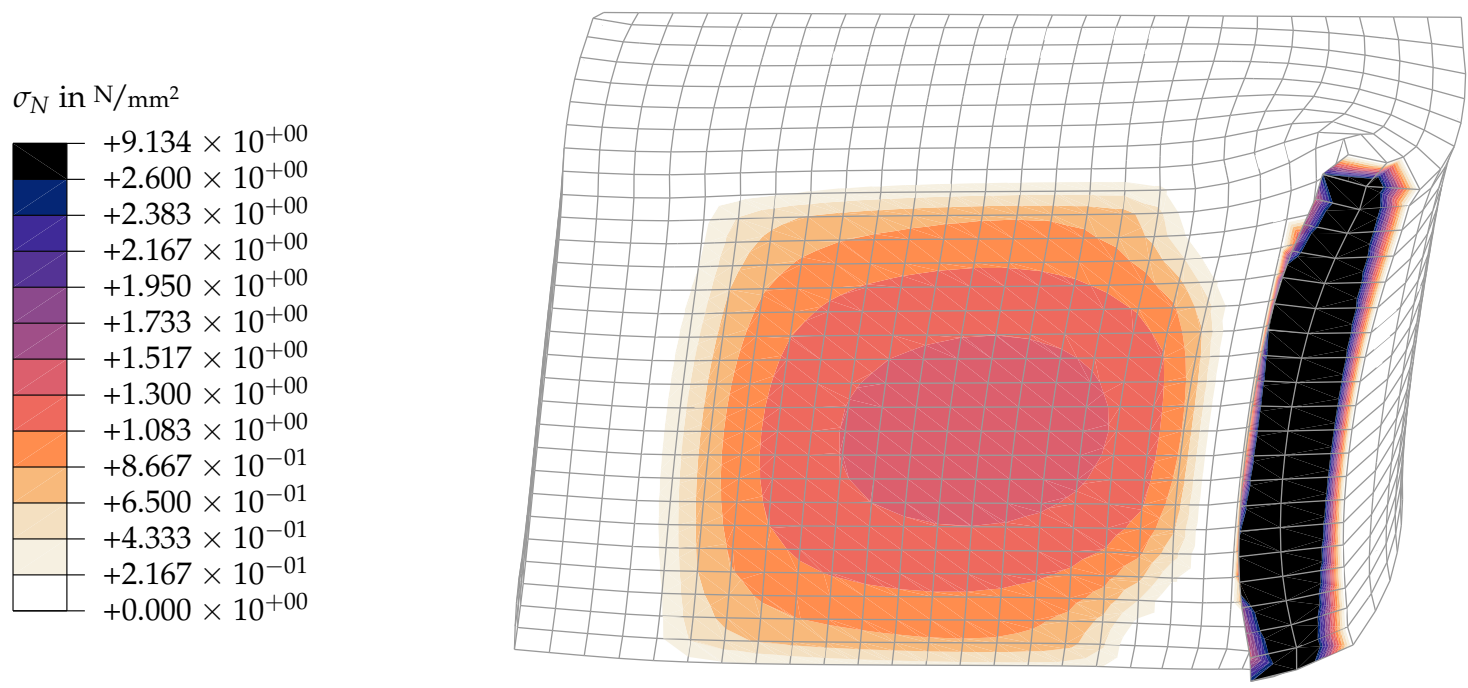

(a)
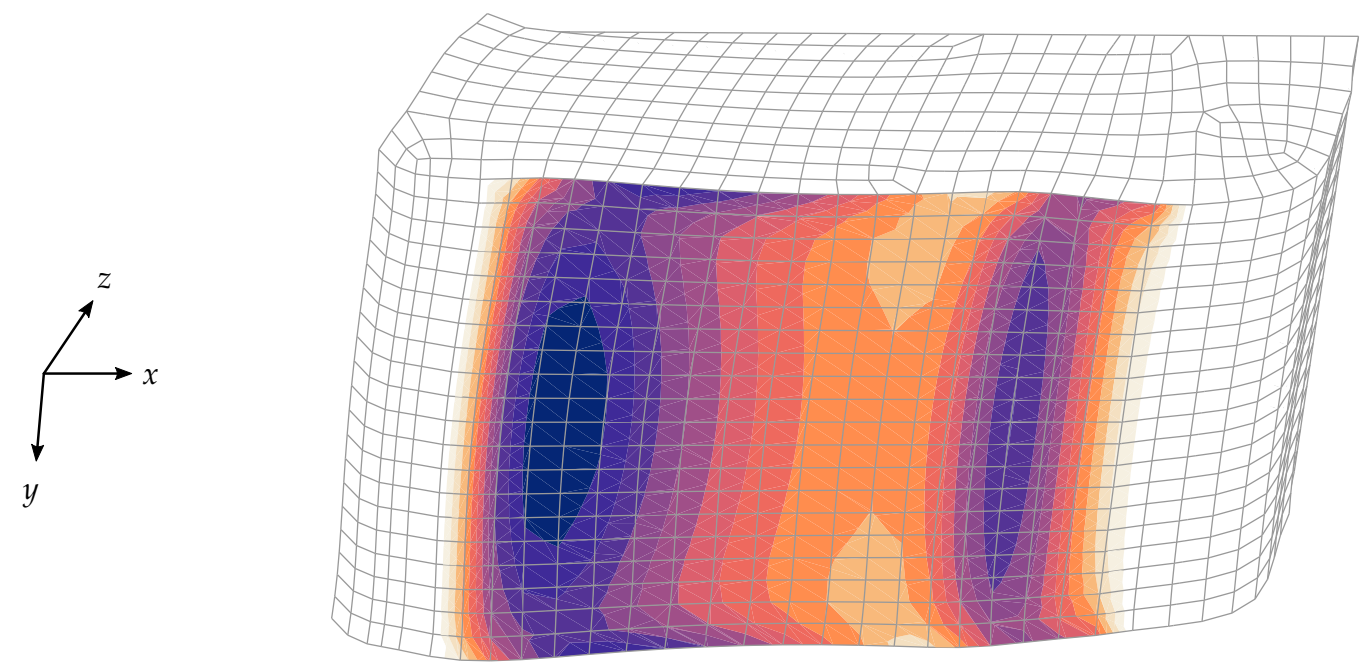

(b)

Figure 17. Contact pressure $\sigma_{N}$ of three-dimensional FEM blocks at $p=1.2 \mathrm{~N} / \mathrm{mm}^{2}$ and $v=100 \mathrm{~mm} / \mathrm{s}$. (a) 4500 elements, unworn, (b) 5120 elements, pre-conditioned. 
The leading edge of the unworn block in Figure 17a folds immediately at the beginning of the sliding step, which causes the simulation to abort. The maximum contact pressure at the leading edge is approximately 10 times larger than contact pressure in the center of the bottom surface and circa five times larger than the largest pressure value in the pre-conditioned block (see Figure 17b). This contact pressure concentration in combination with the abrasive sandpaper leads to uneven wear at the leading edge of the rubber block. The shape of the pre-conditioned sample results as a consequence of this phenomenon.

The different meshes shown in Figures 12 and 13 are analyzed regarding the distribution of the frictional energy rate $\dot{\mathscr{D}}_{s, i}$ (see Equations (47) and (49)) for $i=1, \ldots, n_{n, x}$, where $n_{n, x}$ represents the number of block nodes along the $x$-axis at the bottom surface. To compare the two-dimensional to the three-dimensional models, only the center node line of the bottom surface at $y=0.5 w=10 \mathrm{~mm}$ is used from the three-dimensional block. The element width (expansion in $y$-direction) of the three-dimensional models is $1 \mathrm{~mm}$. In Figure 18, the frictional energy rates per $\mathrm{mm}$ (width) at $p=1.2 \mathrm{~N} / \mathrm{mm}^{2}$ and $v=100 \mathrm{~mm} / \mathrm{s}$ are plotted for the unworn block model with coarse mesh, the preconditioned block model with coarse and fine mesh, as well as the center line of the three-dimensional pre-conditioned block model.

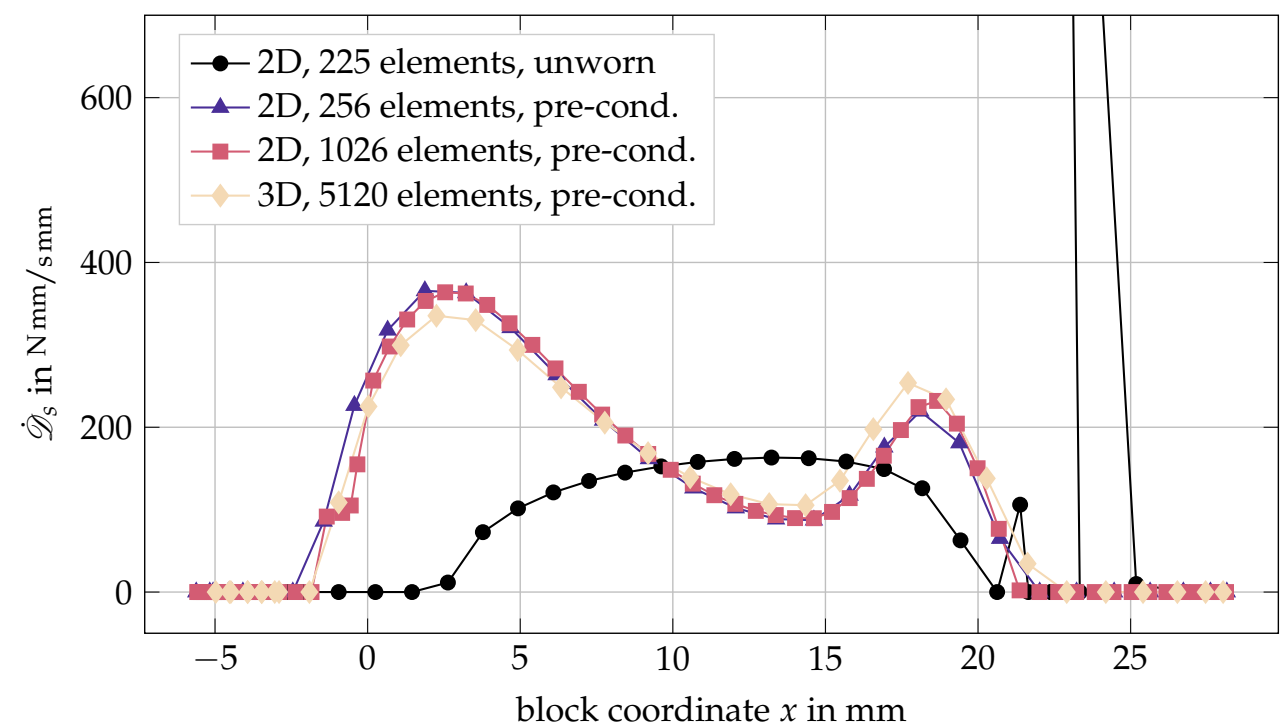

Figure 18. Frictional energy rate distribution $p=1.2 \mathrm{~N} / \mathrm{mm}^{2}$ and $v=100 \mathrm{~mm} / \mathrm{s}$.

The different markers in Figure 18 illustrate the $x$-coordinate of the corresponding block model nodes at the end of the simulation. It can be seen that the contact stress concentration of the unworn block results in a peak value in terms of frictional energy rate in the region of the leading edge $(23 \mathrm{~mm}<x<25 \mathrm{~mm})$. The good agreement of the output of the pre-conditioned block models displayed in Figure 18 proves that the application of two-dimensional models is valid to investigate wear as consequence of frictional energy on block level.

\subsubsection{Verification}

The re-meshing algorithm presented in Section 2.4 is verified at two different test conditions, $p=0.8 \mathrm{~N} / \mathrm{mm}^{2}$ and $v=10 \mathrm{~mm} / \mathrm{s}$ as well as $p=1.2 \mathrm{~N} / \mathrm{mm}^{2}$ and $v=100 \mathrm{~mm} / \mathrm{s}$. For the verification, a linear wear model with its wear coefficient $k_{w}=\dot{V}_{w, \exp } / \dot{\mathscr{D}}_{s, \exp }$ is chosen for the corresponding test conditions (not the coefficients in Table 3 ). The wear coefficients are $k_{w, 1}=2.969 \times 10^{-4} \mathrm{~mm}^{2} / \mathrm{N}$ and $k_{w, 2}=3.712 \times 10^{-4} \mathrm{~mm}^{2} / \mathrm{N}$ for Tests 1 and 2 , respectively. Figure 19 shows the comparison of the experiment, the simulation using the post-processing re-meshing algorithm (PP) and simulation, where the adaptive meshing algorithm is applied (AM) in terms of cumulative mass loss. For this purpose, the volume 
loss is multiplied by the rubber density $\rho=1.12 \times 10^{-3} \mathrm{~g} / \mathrm{mm}^{3}$. Each marker represents one test run.

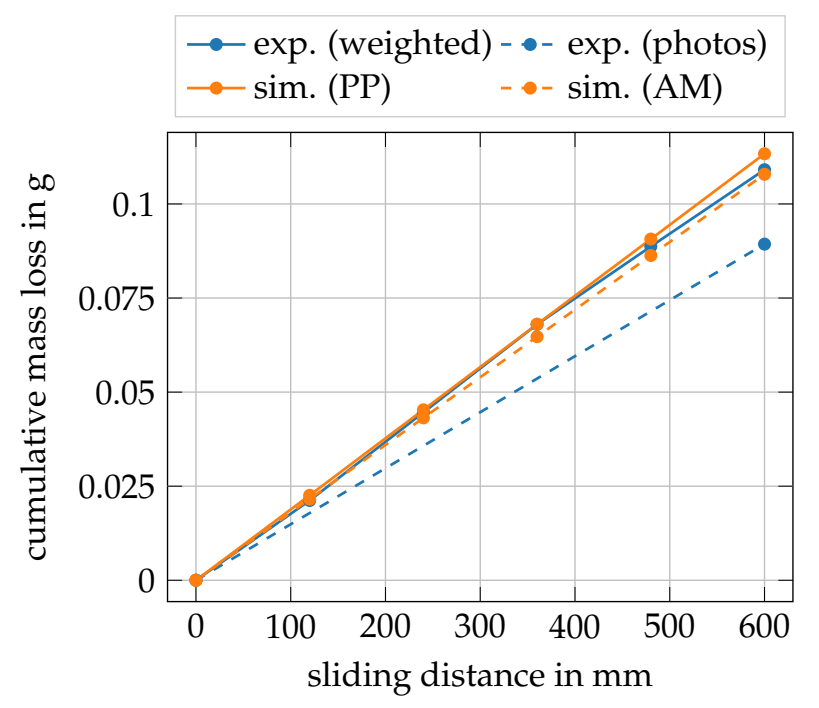

(a)

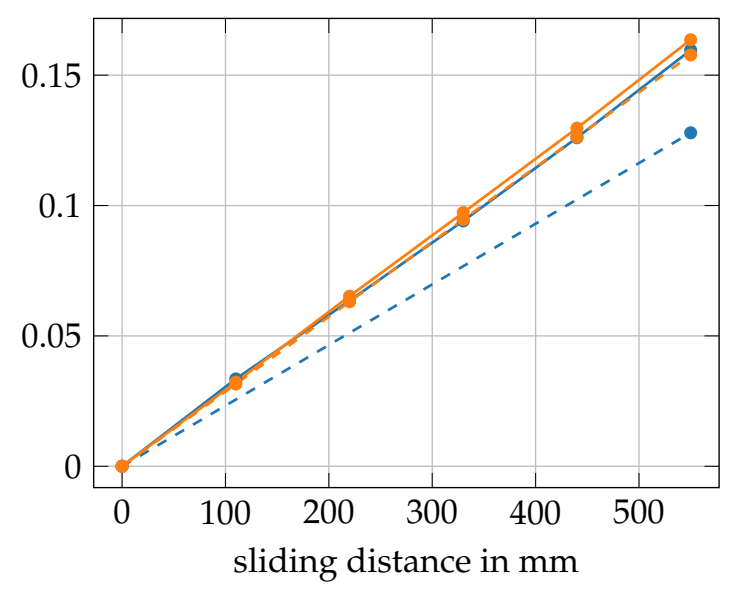

(b)

Figure 19. Comparison of cumulative mass loss. (a) $p=0.8 \mathrm{~N} / \mathrm{mm}^{2}$ and $v=10 \mathrm{~mm} / \mathrm{s}$, (b) $p=1.2 \mathrm{~N} / \mathrm{mm}^{2}$ and $v=100 \mathrm{~mm} / \mathrm{s}$.

Both algorithms can successfully reproduce the expected total wear loss and the application of the nodal area in the reference configuration in Equations (50) and (58) (see Section 2.4) are verified. For the adaptive meshing algorithm, the mesh of the rubber block is updated after each time increment. Hence, the difference between the simulation times of both re-meshing algorithms can be interpreted as maximum computational cost reduction. Regarding the first $\left(p=0.8 \mathrm{~N} / \mathrm{mm}^{2}, v=10 \mathrm{~mm} / \mathrm{s}\right)$ and the second $\left(p=1.2 \mathrm{~N} / \mathrm{mm}^{2}\right.$, $v=100 \mathrm{~mm} / \mathrm{s}$ ) test, the computational time is reduced by $62.4 \%$ and $39.9 \%$, accordingly. In addition, the output of the image processing can be evaluated by computing the area between the contour of the pre-conditioned block and the block after the wear test. This area is multiplied by the block width of $w=20 \mathrm{~mm}$ and the rubber density to get the final mass loss. Both, Figure 19a,b show less wear that results from the photos in comparison to the weighted mass loss during the corresponding linear friction test. There are two possible reasons, which could explain the deviation to the weighted mass loss of approximately 18 to $20 \%$ (Tests 1 and 2). First, the photographs record only the side profile of the blocks, whereby slightly more wear is observed in the center of the rubber sample (see also contact pressure distribution in Figure 17b). Second, the block contour after the wear test often contains some small rubber particles, which are attached to the bottom surface of the block. This reduces the area between the different block contours and leads therefore to less computed mass loss.

\subsubsection{Validation}

To validate both re-meshing algorithms, the final block contours of the simulation are compared to contours, which result from the image processing procedure using the photograph of the worn rubber samples. In the first validation part, the simulation results from the verification are used. Figure 20 illustrates the comparison for the test conditions at $p=0.8 \mathrm{~N} / \mathrm{mm}^{2}$ and $v=10 \mathrm{~mm} / \mathrm{s}$ as well as $p=1.2 \mathrm{~N} / \mathrm{mm}^{2}$ and $v=100 \mathrm{~mm} / \mathrm{s}$. Blue filled circles represent the sections of the final block contour that are aligned to the contour of the pre-conditioned block, because the image processing tool sometimes includes small rubber particles in the final block shape. 


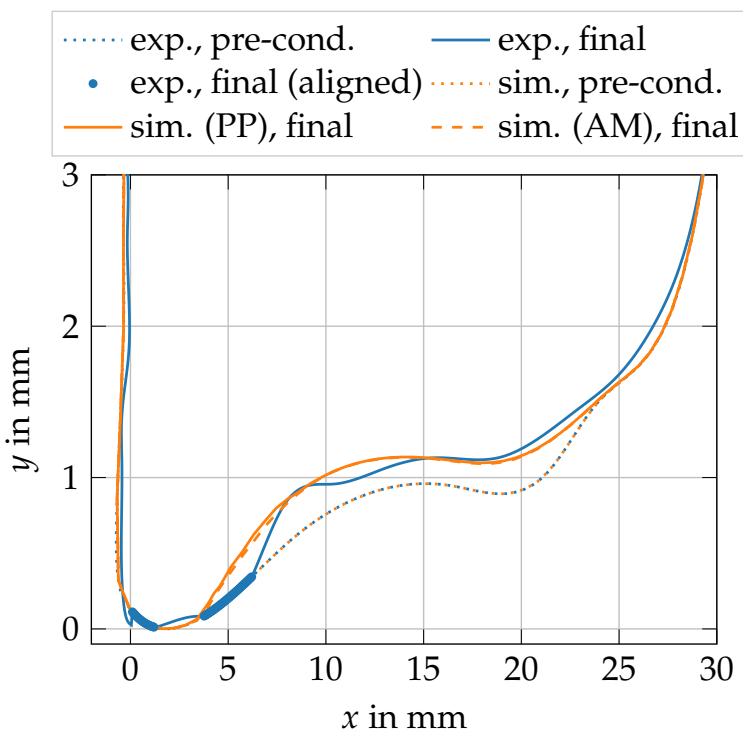

(a)

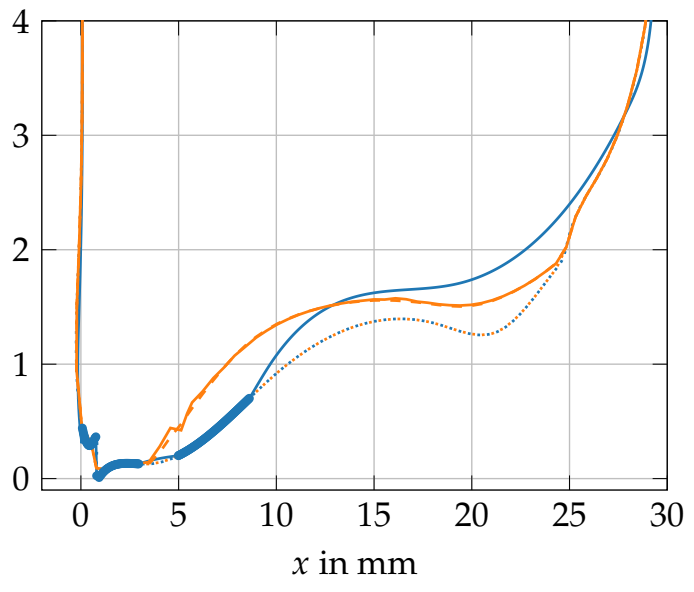

(b)

Figure 20. Comparison of rubber block's contour change. (a) $p=0.8 \mathrm{~N} / \mathrm{mm}^{2}$ and $v=10 \mathrm{~mm} / \mathrm{s}$, (b) $p=1.2 \mathrm{~N} / \mathrm{mm}{ }^{2}$ and $v=100 \mathrm{~mm} / \mathrm{s}$.

Figure 20a shows very good agreement, especially in the center of the bottom surface. However in Figure 20b, the simulated wear loss in the region $5 \mathrm{~mm}<x<10 \mathrm{~mm}$ is significantly larger. In the interval $20 \mathrm{~mm}<x<25 \mathrm{~mm}$, it is vice versa. Most likely, the pre-conditioned block shape is not identified with sufficient accuracy by image processing, which could lead to a deviating contact pressure distribution in the simulations. However, both re-meshing algorithms show almost the same final block contour at the investigated test conditions. For this reason, only the post-processing algorithm is applied for the second part of the validation, because the computational costs are $67 \%$ and $48 \%$ lower for Tests 1 and 2, respectively (averaged over all test runs) in comparison to the adaptive meshing algorithm.

In the second part of the validation, the difference between final contour from recorded photographs versus simulation is analyzed using the identified wear parameters in Table 3 (linear and non-linear with relative error measurement). Figure 21 confirms the result of Figure 20.

The simulated wear close to the trailing block edge exceeds the outcome of the photographs, especially at $p=1.2 \mathrm{~N} / \mathrm{mm}^{2}$ and $v=100 \mathrm{~mm} / \mathrm{s}$. Near the leading block edge, only the simulation of the second test underestimates the mass loss significantly. Note, the difference of mass losses $\Delta m_{w}$ in the legend of Figure 21. The simulations with non-linear wear parameters lead to less wear in comparison to the weighted mass loss in the experiment, although the corresponding markers in Figure 15 are very close to the fitted wear model. The reason is described exemplary for Test $2\left(p=1.2 \mathrm{~N} / \mathrm{mm}^{2}\right.$ and $v=100 \mathrm{~mm} / \mathrm{s}$ ). Figure 18 shows that the maximum frictional energy rate is approximately $360 \mathrm{~N} \mathrm{~mm} / \mathrm{s} \mathrm{mm} \times 20 \mathrm{~mm}=7200 \mathrm{~N} \mathrm{~mm} / \mathrm{s}$. If this value is inserted into the non-linear wear model on the one hand and in the linear wear model with $k_{w, 2}$ on the other, there is already a deviation of $-13.8 \%$ in terms of volume loss. This discrepancy is increased for lower frictional energy rates due to the non-linearity correlation between frictional energy rate and wear loss rate. 


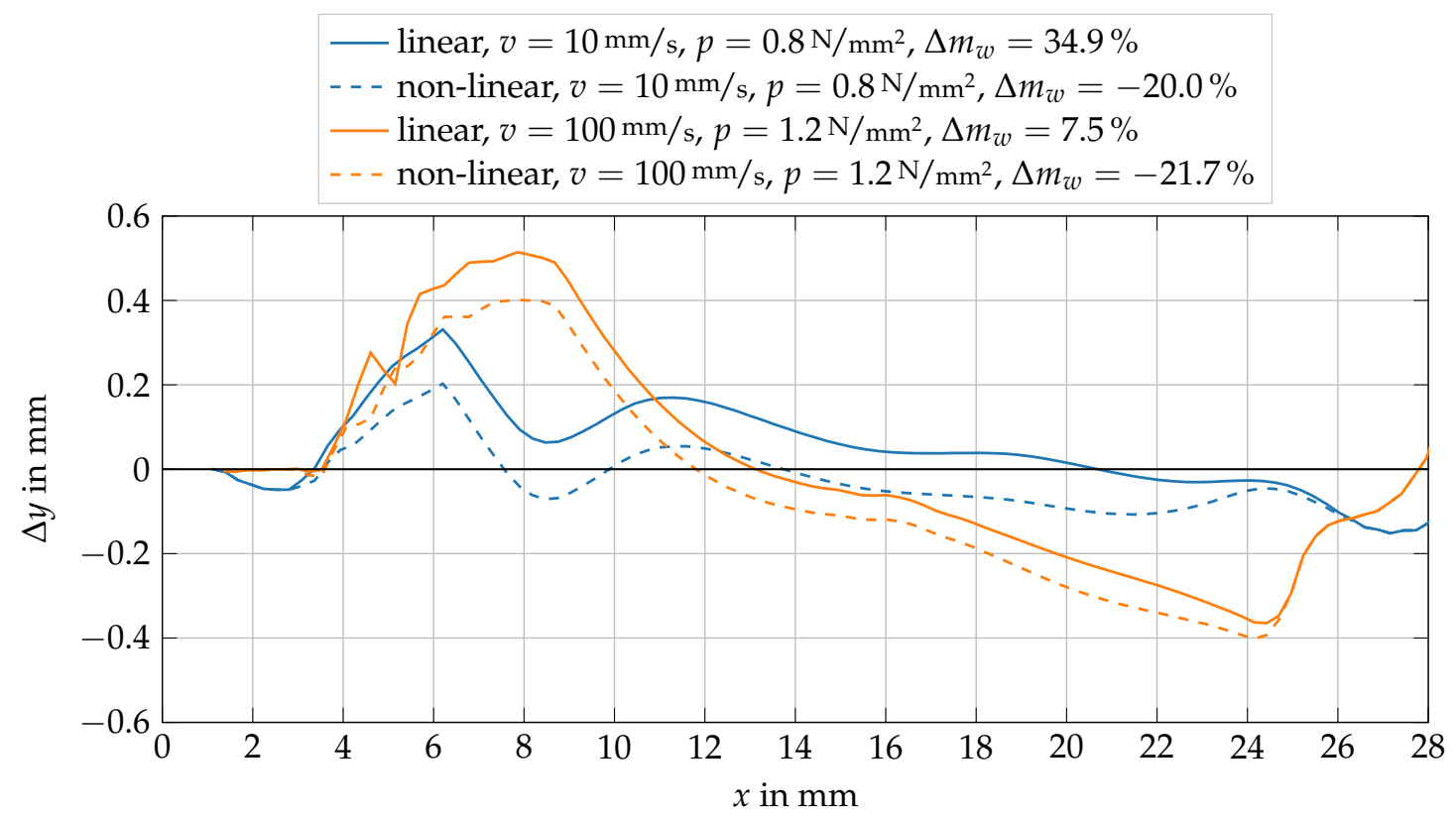

Figure 21. Final block contour difference between experiment and simulation.

\section{Conclusions}

A wear model based on frictional energy rate is proposed in this work. Two different re-meshing algorithms are implemented in order to update the block geometry due to wear. Linear friction and wear tests on block level at different loads and sliding velocities are performed to identify the friction features and wear model parameters. The rubber block samples are run-in (pre-conditioning) until a constant wear rate is observed during the laboratory tests. By recording the change of the longitudinal rubber profile, it is possible to validate the re-meshing algorithm. For this purpose, an image processing tool detects the block shape after pre-conditioning and after the wear test to generate the FE mesh and compare the worn block geometry with the wear simulation results, respectively.

The quality of the photos, especially the lighting conditions, influences the reliability of the image processing tool significantly. In order to compare the block shape from experiments with the wear simulations, the accuracy of the image processing tool should be in $0.01 \mathrm{~mm}$ range. This could not be achieved for all wear tests, because the contrast between background and rubber sample varies within the photo due to non-centralized exposure to light. However, the application of the pre-conditioned block shape is required to obtain a reasonable contact pressure distribution at the bottom surface of the finite element block (see Figure 17). Due to the rate-dependent material properties of rubber, it is necessary to model the entire process of the wear test, starting from rubber compression and acceleration phase on aluminum. A comparison between two- and three-dimensional block models with respect to the evolution of frictional energy proves the usability of twodimensional block models for this type of wear analysis to reduce the computational costs (see Figure 18). A verification study of the linear wear model in combination with both re-meshing algorithms confirms the correct implementation by comparing the resulting mass loss of the wear test with the simulation (see Figure 19). In case of steady-state friction and wear during (short-term) sliding, it is recommended to consider a post-processing remeshing algorithm to update the finite element mesh in order to reduce the computational costs. In general, the worn block model geometry after the wear simulation is in good agreement with the output of the image processing tool. Note that the results of the block shape comparison strongly depends on the photo quality and image processing parameters. 
Author Contributions: The contributions of the authors are listed in the following categories: conceptualization, F.H., M.W. and M.K.; methodology, F.H., M.A.G., M.H. and M.W.; software, F.H., M.A.G. and T.B.; validation, F.H. and M.H.; formal analysis, F.H., M.A.G. and M.H.; investigation, F.H., M.A.G. and M.H.; resources, M.W. and M.K.; data curation, F.H.; writing-original draft preparation, F.H., M.A.G., T.B., M.H. and M.W.; writing-review and editing, F.H., M.W. and M.K.; visualization, F.H. and M.A.G.; supervision, M.W. and M.K.; project administration, M.W. and M.K.; funding acquisition, M.K. All authors have read and agreed to the published version of the manuscript.

Funding: The project has been funded by CEAT Tyres Ltd., India.

Institutional Review Board Statement: Not applicable.

Informed Consent Statement: Not applicable.

Data Availability Statement: The data presented in this study are available on request from the corresponding author.

Acknowledgments: The support of CEAT Tyres Ltd., India, Leibniz Universität Hannover and Technische Universität Dresden is gratefully acknowledged.

Conflicts of Interest: The authors declare no conflict of interest. The funders were involved in the design of the experimental study of friction tests, however, they had no role in the design of the wear and simulation study, in the collection, analyses, or interpretation of data, in the writing of the manuscript, or in the decision to publish the results.

\section{References}

1. Schulze, T.; Bolz, G.; Strübel, C.; Wies, B. Tire technology in target conflict of rolling resistance and wet grip. ATZ Worldw. 2010, 112, 26-32. [CrossRef]

2. Baensch-Baltruschat, B.; Kocher, B.; Stock, F.; Reifferscheid, G. Tyre and road wear particles (TRWP)-A review of generation, properties, emissions, human health risk, ecotoxicity, and fate in the environment. Sci. Total Environ. 2020, 733, 137823. [CrossRef]

3. Nguyen, V.; Zheng, D.; Schmerwitz, F.; Wriggers, P. An advanced abrasion model for tire wear. Wear 2018, 396, 75-85. [CrossRef]

4. Li, Z.; Li, Z.; Wang, Y. An Integrated Approach for Friction and Wear Simulation of Tire Tread Rubber. Part II: Wear Test, Characterization and Modeling. Tire Sci. Technol. 2020, 48, 146-165. [CrossRef]

5. Kahms, S.; Wangenheim, M. Experimental Investigation and Simulation of Aircraft Tire Wear. Tire Sci. Technol. 2021, 49, 55-74. [CrossRef]

6. $\quad$ Pacejka, H. Tire and Vehicle Dynamics, 3rd ed.; Elsevier: Amsterdam, The Netherlands, 2012.

7. Ripka, S.; Gäbel, G.; Wangenheim, M. Dynamics of a siped tire tread block-experiment and simulation. Tire Sci. Technol. 2009, 37, 323-339. [CrossRef]

8. Genovese, A.; D'Angelo, G.A.; Sakhnevych, A.; Farroni, F. Review on friction and wear test rigs: An overview on the state of the art in tyre tread friction evaluation. Lubricants 2020, 8, 91. [CrossRef]

9. Rosu, I.; Elias-Birembaux, H.L.; Lebon, F.; Lind, H.; Wangenheim, M. Experimental and numerical simulation of the dynamic frictional contact between an aircraft tire rubber and a rough surface. Lubricants 2016, 4, 29. [CrossRef]

10. Zheng, D. Prediction of tire tread wear with FEM steady state rolling contact simulation. Tire Sci. Technol. 2003, 31, 189-202. [CrossRef]

11. Hegadekatte, V.; Huber, N.; Kraft, O. Finite element based simulation of dry sliding wear. Model. Simul. Mater. Sci. Eng. 2004, 13, 57. [CrossRef]

12. Runge, S.; Ignatyev, P.; Wangenheim, M.; Bederna, C.; Wies, B.; Wallaschek, J. Transient abrasion on a rubber sample due to highly dynamic contact conditions. Wear 2021, 203848. [CrossRef]

13. Moldenhauer, P.; Kröger, M. Simulation and experimental investigations of the dynamic interaction between tyre tread block and road. In Elastomere Friction: Theory, Experiment and Simulation; Springer: Berlin, Germany, 2010; pp. 165-200. [CrossRef]

14. Kaliske, M.; Rothert, H. Formulation and implementation of three-dimensional viscoelasticity at small and finite strains. Comput. Mech. 1997, 19, 228-239. [CrossRef]

15. Holzapfel, G.A. Nonlinear Solid Mechanics: A Continuum Approach for Engineering; Wiley: Chichester, UK, 2000.

16. Yeoh, O. Characterization of Elastic Properties of Carbon-Black-Filled Rubber Vulcanizates. Rubber Chem. Technol. 1990, 63, 792-805. [CrossRef]

17. Rackl, M. Curve Fitting for Ogden, Yeoh and Polynomial Models. In Proceedings of the 7th International Scilab Users Conference, Paris, France, 21-22 May 2015.

18. Gent, A.; Pulford, C. Mechanisms of Rubber Abrasion. J. Appl. Polym. Sci. 1983, 28, 943-960. [CrossRef]

19. Archard, J.F. Contact and rubbing of flat surfaces. J. Appl. Phys. 1953, 24, 981-988. [CrossRef]

20. Schallamach, A.; Turner, D. The wear of slipping wheels. Wear 1960, 3, 1-25. [CrossRef]

21. Li, Z.; Li, Z.; Wang, Y. An Integrated Approach for Friction and Wear Simulation of Tire Tread Rubber. Part I: Friction Test, Characterization and Modeling. Tire Sci. Technol. 2020, 48, 123-145. [CrossRef] 
22. Wriggers, P. Computational Contact Mechanics; Springer: Berlin, Germany, 2006. [CrossRef]

23. Walt, S.v.d.; Schönberger, J.L.; Nunez-Iglesias, J.; Boulogne, F.; Warner, J.D.; Yager, N.; Gouillart, E.; Yu, T. scikit-image: Image processing in Python. Peer] 2014, 2, e453. [CrossRef]

24. Histogram Equalization with Python and NumPy. Available online: http:/ /www.janeriksolem.net/histogram-equalizationwith-python-and.html (accessed on 23 April 2021).

25. Sobel, I.; Feldman, G. An Isotropic $3 \times 3$ Image Gradient Operator. Presentation at Stanford A.I. Project. 1968. Available online: https://www.researchgate.net/publication/239398674_An_Isotropic_3x3_Image_Gradient_Operator/citation/download (accessed on 23 April 2021).

26. Lorensen, W.E.; Cline, H.E. Marching cubes: A high resolution 3D surface construction algorithm. ACM Siggraph Comput. Graph. 1987, 21, 163-169. [CrossRef]

27. Rublee, E.; Rabaud, V.; Konolige, K.; Bradski, G. ORB: An efficient alternative to SIFT or SURF. In Proceedings of the 2011 International Conference on Computer Vision, Barcelona, Spain, 6-13 November 2011; pp. 2564-2571. [CrossRef]

28. Fischler, M.A.; Bolles, R.C. Random sample consensus: A paradigm for model fitting with applications to image analysis and automated cartography. Commun. Assoc. Comput. Mach. 1981, 24, 381-395. [CrossRef]

29. Dierckx, P. Algorithms for smoothing data with periodic and parametric splines. Comput. Graph. Image Process. 1982, 20, 171-184. [CrossRef]

30. Simo, J.; Laursen, T. An augmented lagrangian treatment of contact problems involving friction. Comput. Struct. 1992, 42, 97-116. [CrossRef] 